\title{
BOUNDARY REGULAR FIXED POINTS IN LOEWNER THEORY
}

\author{
FILIPPO BRACCI*, MANUEL D. CONTRERAS ${ }^{\dagger}$, SANTIAGO DÍAZ-MADRIGAL $^{\dagger}$, \\ AND PAVEL GUMENYUK ${ }^{\ddagger}$
}

\begin{abstract}
We characterize regular fixed points of evolution families in terms of analytical properties of the associated Herglotz vector fields and geometrical properties of the associated Loewner chains. We present several examples showing the rôle of the given conditions. Moreover, we study the relations between evolution families and Herglotz vector fields at regular contact points and prove an embedding result for univalent selfmaps of the unit disc with a given boundary regular fixed point into an evolution family with prescribed boundary data.
\end{abstract}

\section{INTRODUCTION}

Loewner theory, which originated in Ch. Loewner's seminal paper [27] of 1923 and later was developed deeply by P.P. Kufarev [26] and Ch. Pommerenke [28], [29, Chapter 6], is nowadays one of the main tools in geometric function theory. Loewner Theory proved to be effective in many extremal problems for univalent functions hardly accessible with other methods. The most famous example is its crucial rôle in the proof of the Bieberbach conjecture given by L. de Branges. Recently many mathematicians have studied a stochastic variant of the Loewner equation (SLE) introduced by O. Schramm. This leads to a breakthrough in several problems of statistical physics and probability theory. A historical overview and bibliography on Loewner Theory can be found, e.g., in survey papers $[2,7]$.

More recently, the authors of this paper have developed a general Loewner theory using an approach, which is different from the classical one and which extends also to complex

Date: June 21, 2018.

2010 Mathematics Subject Classification. Primary 37C10, 30C35; Secondary 30D05, 30C80, 37F99, $37 \mathrm{C} 25$.

Key words and phrases. Loewner chain, evolution family, boundary fixed point, univalent function, conformal mapping.

* Partially supported by the ERC grant "HEVO - Holomorphic Evolution Equations" n. 277691.

† Partially supported by the Ministerio de Economía y Competitividad and the European Union (FEDER), projects MTM2009-14694-C02-02 and MTM2012-37436-C02-01, and by La Consejería de Educación y Ciencia de la Junta de Andalucía.

$\ddagger$ Partially supported by the FIRB grant Futuro in Ricerca "Geometria Differenziale Complessa e Dinamica Olomorfa" n. RBFR08B2HY. 
hyperbolic manifolds $[8,9,14,5]$. Note that an extension of the classical Loewner theory to several complex variables had been treated for a long time, see, e.g., [24].

According to the new approach, Loewner theory relates three objects: Herglotz vector fields, evolution families and Loewner chains. Roughly speaking, a Herglotz vector field $G(z, t)$ is a Carathéodory vector field such that $G(\cdot, t)$ is semicomplete for almost every $t \geq 0$. An evolution family $\left(\varphi_{s, t}\right)$ is a family of holomorphic self-maps of the unit disc $\mathbb{D}$ satisfying a kind of semigroup-type algebraic relations and some regularity hypotheses in $s$ and $t$. Finally, a Loewner chain $\left(f_{t}\right)$ is a family of univalent mappings of the unit disc with increasing ranges satisfying a certain regularity assumption in $t$. See Section 2.3 for precise definitions and basic results.

This three objects are related by the following fundamental equations:

$$
\frac{\partial \varphi_{s, t}(z)}{\partial t}=G\left(\varphi_{s, t}(z), t\right), \quad \frac{\partial f_{t}(z)}{\partial t}=-f_{t}^{\prime}(z) G(z, t), \quad f_{s}(z)=f_{t}\left(\varphi_{s, t}(z)\right) .
$$

The aim of the present paper is to study the boundary behavior of the three objects, relating dynamical properties of evolution families with analytical properties of the corresponding Herglotz vector fields and (in some cases) geometrical properties of Loewner chains.

In order to set up our results, we need to introduce some notations and definitions. Following [30, §4.3], if $f: \mathbb{D} \rightarrow \mathbb{C}$ is holomorphic and $\sigma \in \mathbb{T}:=\partial \mathbb{D}$ we say that $f$ is conformal at $\sigma$ if the non-tangential limit of $f$ at $\sigma$ exists - and we denote it $f(\sigma)$-and the non-tangential limit of the incremental ratio of $f$ at $\sigma$ exists finitely and different from 0 . Let $\left(\varphi_{s, t}\right)$ be an evolution family in $\mathbb{D}$. A point $\sigma \in \mathbb{T}$ is a boundary regular fixed point of $\left(\varphi_{s, t}\right)$ if $\varphi_{s, t}$ is conformal at $\sigma$ and $\varphi_{s, t}(\sigma)=\sigma$ for all $t \geq s \geq 0$. The spectral function of $\left(\varphi_{s, t}\right)$ at a boundary regular fixed point $\sigma \in \mathbb{T}$ is $\Lambda:[0,+\infty) \rightarrow \mathbb{R}$ defined by $\Lambda(t):=-\log \left|\varphi_{0, t}^{\prime}(\sigma)\right|$. We prove that such a function is of bounded variation.

A boundary regular null point for a holomorphic vector field $H: \mathbb{D} \rightarrow \mathbb{C}$ is a point $\sigma \in \mathbb{T}$ such that $H$ has a non-tangential singularity at $\sigma$ and the non-tangential limit of the incremental ratio of $H$ exists finitely at $\sigma$.

Our main result is the following:

Theorem 1.1. Let $\left(\varphi_{s, t}\right)$ be an evolution family of order $d \in[1,+\infty]$, let $G$ be its Herglotz vector field, and let $\sigma \in \mathbb{T}$. The following assertions are equivalent:

(A) for each $t \geq s \geq 0$ the point $\sigma$ is a boundary regular fixed point of $\left(\varphi_{s, t}\right)$;

(B) The Herglotz vector field $G$ satisfies the following two conditions:

(B.1) for a.e. $t \geq 0, G(\cdot, t)$ has a boundary regular null point at $\sigma$;

(B.2) the function $t \mapsto G^{\prime}(\sigma, t)$ is locally integrable in $[0,+\infty)$.

Moreover, if one (and hence both) of these assertions holds, then the spectral function $\Lambda$ of $\left(\varphi_{s, t}\right)$ at $\sigma$ satisfies

$$
\Lambda(t)=-\int_{0}^{t} G^{\prime}(\sigma, s) d s \quad \text { for all } t \geq 0 .
$$


Furthermore, let $\left(f_{t}\right)$ be a Loewner chain associated with $\left(\varphi_{s, t}\right)$ and suppose there exists $t_{0} \geq 0$ such that the map $f_{t_{0}}$ is conformal at $\sigma$. Then $(A)$ and $(B)$ are equivalent to:

(C) for every $s \geq 0$ the following assertions hold:

(C.1) the map $f_{s}$ is conformal at $\sigma$;

(C.2) $f_{s}(\sigma)=f_{t_{0}}(\sigma)$;

(C.3) $\limsup _{t \rightarrow s^{+}}\left|\arg \left(f_{t}^{\prime}(\sigma) / f_{s}^{\prime}(\sigma)\right)\right|<\pi$.

Moreover, if condition $(C)$ holds, then the function $t \mapsto f_{t}^{\prime}(\sigma)$ is locally absolutely continuous on $[0,+\infty)$, with $\arg f_{t}^{\prime}(\sigma)$ being constant.

The proof is given in Section 4. In Corollary 6.1 we show that a similar result holds if $f_{t_{0}}$ has a simple pole at $\sigma$ and $(\mathrm{C})$ is replaced with suitable conditions for this case. In Section 6 we present examples showing that conditions (B.1) and (C.3) cannot be omitted, and explain the essential role of the conformality of $f_{t_{0}}$ at $\sigma$. Moreover, several (counter)examples to natural conjectures concerning the regularity of $t \mapsto G^{\prime}(\sigma, t)$ versus the $L^{d}$-regularity of the evolution family are also given.

In part, Theorem 1.1 is a consequence of a more general result on regular contact points of evolution families, which relates them with analytic behavior of Herglotz vector fields, see Theorem 3.5.

Finally, as an application of our main result, in Section 5 we prove the following embedding theorem with prescribed boundary data:

Theorem 1.2. Suppose $\phi \in \operatorname{Hol}(\mathbb{D}, \mathbb{D})$ is univalent and has a boundary regular fixed point at $\sigma \in \mathbb{T}$. Then for any $t_{0}>0$ and for any locally absolutely continuous function $\Lambda:[0,+\infty) \rightarrow \mathbb{R}$ with $\Lambda(0)=0$ and $\Lambda\left(t_{0}\right)=-\log \phi^{\prime}(\sigma)$ there exists an evolution family $\left(\varphi_{s, t}\right)$ satisfying the following conditions:

(i) $\left(\varphi_{s, t}\right)$ has a boundary regular fixed point at $\sigma$,

(ii) the spectral function of $\left(\varphi_{s, t}\right)$ at $\sigma$ coincides with $\Lambda$,

(iii) $\varphi_{0, t_{0}}=\phi$.

\section{Preliminaries}

2.1. Boundary regular contact and fixed points. In what follows, for a map $f: \mathbb{D} \rightarrow \mathbb{C}$ and a point $\sigma \in \mathbb{T}:=\partial \mathbb{D}$, we denote by $\angle \lim _{z \rightarrow \sigma} f(z)$ the angular (or non-tangential) limit of $f$ at $\sigma$.

$\operatorname{By} \operatorname{Hol}(U, W)$ we will denote the class of all holomorphic maps of $U$ into $W$.

Definition 2.1. Let $\varphi \in \operatorname{Hol}(\mathbb{D}, \mathbb{D})$. A point $\sigma \in \mathbb{T}$ is called a contact point if the angular limit $\varphi(\sigma):=\angle \lim _{z \rightarrow \sigma} \varphi(z)$ exists and belongs to $\mathbb{T}$. If, in addition, the angular derivative

$$
\varphi^{\prime}(\sigma):=\angle \lim _{z \rightarrow \sigma} \frac{\varphi(z)-\varphi(\sigma)}{z-\sigma}
$$

exists finitely, then the contact point $\sigma$ is said to be regular. 
A (regular) contact point $\sigma$ for $\varphi \in \operatorname{Hol}(\mathbb{D}, \mathbb{D})$ is called a boundary (regular) fixed point if $\varphi(\sigma)=\sigma$. For shortness, we will write "BRFP" for "boundary regular fixed point".

A characterization of regular contact points is given by the classical Julia-WolffCarathéodory theorem (see, e.g., $[1, \S 1.2 .1]$ or $[3$, p. $7-12]$ ). By the boundary dilatation coefficient of $\varphi \in \operatorname{Hol}(\mathbb{D}, \mathbb{D})$ at $\sigma \in \mathbb{T}$ we mean

$$
\alpha_{\varphi}(\sigma):=\liminf _{z \rightarrow \sigma} \frac{1-|\varphi(z)|}{1-|z|} .
$$

Theorem 2.2 (Julia-Wolff-Carathéodory). Let $\varphi \in \operatorname{Hol}(\mathbb{D}, \mathbb{D})$ and $\sigma \in \mathbb{T}$. Then the following four statements are equivalent:

(i) $\sigma$ is a regular contact point of $\varphi$;

(ii) $\alpha_{\varphi}(\sigma)<+\infty$;

(iii) there exist a point $\omega \in \mathbb{T}$ and $A>0$ such that for all $z \in \mathbb{D}$

$$
\frac{|\omega-\varphi(z)|^{2}}{1-|\varphi(z)|^{2}} \leq A \frac{|\sigma-z|^{2}}{1-|z|^{2}}
$$

(iv) $\lim \sup _{(0,1) \ni r \rightarrow 1}|\varphi(r \sigma)|=1$ and $\lim \sup _{(0,1) \ni r \rightarrow 1}\left|\varphi^{\prime}(r \sigma)\right|<+\infty$.

Moreover, if the above conditions hold, then:

(v) the point $\omega$ in (iii) is unique and coincides with $\varphi(\sigma):=\angle \lim _{z \rightarrow \sigma} \varphi(z)$;

(vi) $\varphi^{\prime}(\sigma)=\angle \lim _{z \rightarrow \sigma} \varphi^{\prime}(z)$ and $\sigma \bar{\omega} \varphi^{\prime}(\sigma)=\alpha_{\varphi}(\sigma)=A_{0}$, where $A_{0}$ is the least constant $A$ for which (iii) holds.

Denote by $\operatorname{Möb}(\mathbb{D})$ the Möbius group of all conformal automorphisms of $\mathbb{D}$. The classical theorem of Denjoy - Wolff (see, e.g. $[1, \S 1.3 .2]$ ) can be stated as follows.

Theorem 2.3 (Denjoy-Wolff). Let $\varphi \in \operatorname{Hol}(\mathbb{D}, \mathbb{D}), \varphi \neq \mathrm{id}_{\mathbb{D}}$. Then one of the following alternatives takes place:

(i) $\varphi$ is conjugated to a rotation, i. e., $\varphi \in \operatorname{Möb}(\mathbb{D})$ and has a unique fixed point $\tau \in \mathbb{D}$;

(ii) the sequence of iterates $\left(\varphi^{\circ n}\right)$ converges uniformly on compacta to a unique fixed point $\tau \in \mathbb{D}$ of $\varphi$;

(iii) $\varphi$ has no fixed points in $\mathbb{D}$, but the sequence of iterates $\left(\varphi^{\circ n}\right)$ converges uniformly on compacta to a BRFP $\tau \in \mathbb{T}$ of $\varphi$ with $\alpha_{\varphi}(\tau) \leq 1$.

Moreover, if $\sigma \neq \tau$ is a contact point of $\varphi$, then $\alpha_{\varphi}(\sigma) \geq|1-\bar{\tau} \varphi(\sigma)|^{2} /|1-\bar{\tau} \sigma|^{2}$. The equality occurs only in case (i).

The point $\tau$ is called the Denjoy - Wolff point of $\varphi$ (or, abbreviated, the $D W$-point).

2.2. Semigroups and infinitesimal generators. A semigroup $\left(\phi_{t}\right)$ of holomorphic selfmaps of $\mathbb{D}$ is a continuous homomorphism between the additive semigroup $\left(\mathbb{R}^{+},+\right)$of positive real numbers and the semigroup $(\operatorname{Hol}(\mathbb{D}, \mathbb{D}), \circ)$ of holomorphic self-maps of $\mathbb{D}$ 
with respect to the composition, endowed with the topology of uniform convergence on compacta.

By Berkson - Porta's theorem [6], if $\left(\phi_{t}\right)$ is a semigroup in $\operatorname{Hol}(\mathbb{D}, \mathbb{D})$ then $t \mapsto \phi_{t}(z)$ is analytic and there exists a unique holomorphic vector field $G: \mathbb{D} \rightarrow \mathbb{C}$ such that

$$
\frac{\partial \phi_{t}(z)}{\partial t}=G\left(\phi_{t}(z)\right)
$$

This vector field $G$, called the infinitesimal generator of $\left(\phi_{t}\right)$, is semicomplete in the sense that the Cauchy problem

$$
\left\{\begin{array}{l}
\dot{w}=G(w(t)), \\
w(0)=z,
\end{array}\right.
$$

has a solution $w^{z}:[0,+\infty) \rightarrow \mathbb{D}$ for any $z \in \mathbb{D}$. Conversely, any semicomplete holomorphic vector field in $\mathbb{D}$ generates a semigroup in $\operatorname{Hol}(\mathbb{D}, \mathbb{D})$.

Let $G \not \equiv 0$ be the infinitesimal generator of a one-parameter semigroup $\left(\phi_{t}\right)$. Then there exists a unique $\tau \in \overline{\mathbb{D}}$ and a unique holomorphic $p: \mathbb{D} \rightarrow \mathbb{C}$ with $\operatorname{Re} p(z) \geq 0$ such that the following formula, known as the Berkson-Porta formula, holds

$$
G(z)=(z-\tau)(\bar{\tau} z-1) p(z) .
$$

The point $\tau$ in the Berkson - Porta formula turns out to be the DW-point of all $\phi_{t}$ 's different from $\mathrm{id}_{\mathbb{D}}$. Moreover, if $\tau \in \partial \mathbb{D}$, then $\phi_{t}^{\prime}(\tau)=e^{\beta t}$ for some $\beta \leq 0$, see [35, Theorem (1.7) on p. 19].

Definition 2.4. A boundary regular fixed point of a semigroup $\left(\phi_{t}\right)$ is a point $\sigma \in \partial \mathbb{D}$ which is a boundary regular fixed point of $\phi_{t}$ for any $t \geq 0$.

Remark 2.5. In fact, the condition in the above definition can be replaced by the weaker assumption that $\sigma$ is a BRFP for some $\phi_{t} \neq \mathrm{id}_{\mathbb{D}}$, see $[18$, Theorems 1 and 5].

Definition 2.6. A boundary regular null point (abbreviated, BRNP) of an infinitesimal generator $G$ is a point $\sigma \in \mathbb{T}:=\partial \mathbb{D}$ such that

$$
G^{\prime}(\sigma):=\angle \lim _{z \rightarrow \sigma} \frac{G(z)}{z-\sigma}
$$

exists finitely. The number $G^{\prime}(\sigma)$ is called the dilation of $G$ at $\sigma$.

Remark 2.7. The number $G^{\prime}(\sigma)$ in the above definition is always real, see [19, Theorem 1].

In the following theorem we collect some known results concerning infinitesimal generators of one-parameter semigroups with a BRFP at a given point $\sigma \in \mathbb{T}$. By $\mathcal{P}$ we denote the class of all $p \in \operatorname{Hol}(\mathbb{D}, \mathbb{C})$ such that $\operatorname{Re} p(z) \geq 0$ for all $z \in \mathbb{D}$.

Theorem 2.8. Let $\left(\phi_{t}\right)$ be a one-parameter semigroup in $\mathrm{Hol}(\mathbb{D}, \mathbb{D})$ and $G$ its infinitesimal generator. Let $\sigma \in \mathbb{T}$ and $\lambda \in \mathbb{R}$. Then the following statements are equivalent:

(i) for each $t \geq 0$, the function $\phi_{t}$ has a BRFP at $\sigma$ with $\phi_{t}^{\prime}(\sigma)=e^{\lambda t}$; 
(ii) $G$ has a BRNP at $\sigma$ of dilation $G^{\prime}(\sigma)=\lambda$;

(iii) there exits a function $p \in \mathcal{P}$ such that $\angle \lim _{z \rightarrow \sigma}(z-\sigma) p(z)=0$ and

$$
G(z)=(z-\sigma)(\bar{\sigma} z-1)\left(p(z)-\frac{\lambda}{2} \frac{\sigma+z}{\sigma-z}\right) \quad \text { for all } z \in \mathbb{D} .
$$

Remark 2.9. The equivalence between (i) and (ii) is from [18, Theorem 1], [19, Theorem 2], see also [21]. The Berkson - Porta type representation at BRNP given by the equivalence between (iii) and (ii) is in [11] (see also [34]). An analogous representation taking into account the position of the DW-point (which is assumed in this case to be different from the considered BRFP) has been recently given by Goryainov and Kudryavtseva [23].

Lemma 2.10. Let $G$ be an infinitesimal generator. Suppose that $G$ has a BRNP at $\sigma \in \mathbb{T}$ with dilation $\lambda$. Then for all $z \in \mathbb{D}$,

$$
|G(z)| \leq 4\left(\sqrt{2}|G(0)|+(\sqrt{2}+1) \frac{|\lambda|}{2}\right) \frac{|\sigma-z|^{2}}{1-|z|^{2}} .
$$

Proof. Use representation (2.3) in Theorem 2.8. Then $G(0)=\sigma(p(0)-\lambda / 2)$ and hence $|p(0)| \leq|G(0)|+|\lambda| / 2$. Furthermore, $|G(z)| \leq|\sigma-z|^{2}\left(|p(z)|+\left|\frac{\lambda}{2} \frac{\sigma+z}{\sigma-z}\right|\right)$ for all $z \in \mathbb{D}$, which being combined with the growth estimates for the class $\mathcal{P}$ (see, e.g. [29, inequality (11) on p. 40]) results in

$$
|G(z)| \leq|z-\sigma|^{2}\left(\sqrt{2}(|G(0)|+|\lambda| / 2) \frac{1+|z|}{1-|z|}+\frac{|\lambda|}{2} \frac{1+|z|}{1-|z|}\right) .
$$

Taking into account that $\frac{1+|z|}{1-|z|} \leq 4 /\left(1-|z|^{2}\right)$, one easily obtains (2.4).

2.3. Evolution families and generalized Loewner - Kufarev equation. The three main objects of the generalized Loewner theory are Herglotz vector fields, evolution families and Loewner chains (see [8] and [14]).

Definition 2.11. Let $d \in[1,+\infty]$. A Herglotz vector field of order $d$ on the unit disc $\mathbb{D}$ is a function $G: \mathbb{D} \times[0,+\infty) \rightarrow \mathbb{C}$ with the following properties:

H1. For all $z \in \mathbb{D}$, the function $[0,+\infty) \ni t \mapsto G(z, t)$ is measurable;

H2. For all $t \in[0,+\infty)$, the function $\mathbb{D} \ni z \mapsto G(z, t)$ is holomorphic;

H3. For any compact set $K \subset \mathbb{D}$ and for all $T>0$ there exists a non-negative function $k_{K, T} \in L^{d}([0, T], \mathbb{R})$ such that $|G(z, t)| \leq k_{K, T}(t)$ for all $z \in K$ and for almost every $t \in[0, T]$.

H4. For almost every $t \in[0,+\infty), G(\cdot, t)$ is an infinitesimal generator.

In $[8$, Theorem 4.8] it is proved that any Herglotz vector field $G(z, t)$ has an essentially unique representation by means of a Berkson-Porta type formula, namely, $G(z, t)=$ $(z-\tau(t))(\overline{\tau(t)} z-1) p(z, t)$, where $\tau:[0,+\infty) \rightarrow \overline{\mathbb{D}}$ is a measurable function and $p: \mathbb{D} \times$ $[0,+\infty) \rightarrow \mathbb{C}$ has the property that for all $z \in \mathbb{D}$, the function $[0,+\infty) \ni t \mapsto p(z, t) \in \mathbb{C}$ 
belongs to $L_{\text {loc }}^{d}([0,+\infty), \mathbb{C})$; for all $t \in[0,+\infty)$, the function $\mathbb{D} \ni z \mapsto p(z, t) \in \mathbb{C}$ is holomorphic; for all $z \in \mathbb{D}$ and for all $t \in[0,+\infty)$, we have $\operatorname{Re} p(z, t) \geq 0$.

Definition 2.12. Let $d \in[1,+\infty]$. A family $\left(\varphi_{s, t}\right)_{0 \leq s \leq t<+\infty}$ of holomorphic self-maps of the unit disc is called an evolution family of order $d$ if it satisfies the following conditions:

EF1. $\varphi_{s, s}=\mathrm{id}$ for any $s \geq 0$;

EF2. $\varphi_{s, t}=\varphi_{u, t} \circ \varphi_{s, u}$ whenever $0 \leq s \leq u \leq t<+\infty$;

EF3. for any $z \in \mathbb{D}$ and any $T>0$ there exists a non-negative function $k_{z, T} \in$ $L^{d}([0, T], \mathbb{R})$ such that

$$
\left|\varphi_{s, u}(z)-\varphi_{s, t}(z)\right| \leq \int_{u}^{t} k_{z, T}(\xi) d \xi
$$

whenever $0 \leq s \leq u \leq t \leq T$.

The elements of evolution families are univalent [8, Corollary 6.3].

In $[8$, Theorem 1.1, Theorem 6.6] it is proved that there is a one-to-one correspondence between evolution families and Herglotz vector fields:

Theorem 2.13. For any evolution family $\left(\varphi_{s, t}\right)$ of order $d \in[1,+\infty]$ there exists a unique (up to changing on a set of measure zero in $t$ ) Herglotz vector field $G(z, t)$ of order $d$ such that for all $z \in \mathbb{D}$

$$
\frac{\partial \varphi_{s, t}(z)}{\partial t}=G\left(\varphi_{s, t}(z), t\right) \quad \text { for a.e. } t \in[0,+\infty) .
$$

Conversely, for any Herglotz vector field $G(z, t)$ of order $d \in[1,+\infty]$ there exists a unique evolution family $\left(\varphi_{s, t}\right)$ of orderd such that (2.5) is satisfied.

Definition 2.14. Let $d \in[1,+\infty]$. A family $\left(f_{t}\right)_{0 \leq t<+\infty}$ of holomorphic maps of the unit disc is called a Loewner chain of order $d$, if the following conditions hold:

LC1. $f_{t}: \mathbb{D} \rightarrow \mathbb{C}$ is univalent for all $t \geq 0$,

LC2. $f_{s}(\mathbb{D}) \subset f_{t}(\mathbb{D})$ whenever $0 \leq s<t<+\infty$,

LC3. for any compact set $K \subset \mathbb{D}$ and any $T>0$ there exists a non-negative function $k_{K, T} \in L^{d}([0, T], \mathbb{R})$ such that

$$
\left|f_{s}(z)-f_{t}(z)\right| \leq \int_{s}^{t} k_{K, T}(\xi) d \xi
$$

whenever $z \in K$ and $0 \leq s \leq t \leq T$.

Definition 2.15. A Loewner chain $\left(f_{t}\right)$ is said to be associated with an evolution family $\left(\varphi_{s, t}\right)$ if

$$
\varphi_{s, t}=f_{t}^{-1} \circ f_{s} \quad \text { for any } t \geq s \geq 0 .
$$

In [14, Theorem 1.3, Theorem 4.1], see also [17, Section 2.1], it is proved

Theorem 2.16. Let $d \in[1,+\infty]$. The following statements hold: 
(1) For any Loewner chain $\left(f_{t}\right)$ of order $d$ the formula (2.6) defines an evolution fam$i l y\left(\varphi_{s, t}\right)$ of the same order $d$. Conversely, for any evolution family $\left(\varphi_{s, t}\right)$ of order $d$, there exists a Loewner chain $\left(f_{t}\right)$ of order $d$ associated with it.

(2) Let $G(z, t)$ be the Herglotz vector field of an evolution family $\left(\varphi_{s, t}\right)$ of order $d$, and let $(z, t) \mapsto f_{t}(z)$ be a solution to

$$
\frac{\partial f_{s}(z)}{\partial s}=-G(z, s) f_{s}^{\prime}(z)
$$

on $\mathbb{D} \times[0,+\infty)$. Suppose that $f_{t}$ is univalent in $\mathbb{D}$ for every $t \geq 0$. Then $\left(f_{t}\right)$ is a Loewner chain of order $d$ associated with the evolution family $\left(\varphi_{s, t}\right)$.

\section{Spectral FUnCtions AND REgUlar CONTACT POINTS OF EVOLUTiOn FAMilies}

Definition 3.1. Let $\left(\varphi_{s, t}\right)$ be an evolution family of order $d \in[1,+\infty]$. A point $\sigma \in \mathbb{T}$ is called a regular contact point of $\left(\varphi_{s, t}\right)$ if $\sigma$ is a regular contact point of the function $\varphi_{0, t}$ for any $t \geq 0$. The spectral function of $\left(\varphi_{s, t}\right)$ at a regular contact point $\sigma \in \mathbb{T}$ is $\Lambda:[0,+\infty) \rightarrow \mathbb{R}$ defined by $\Lambda(t):=-\log \left|\varphi_{0, t}^{\prime}(\sigma)\right|$.

Remark 3.2. From [19, Lemma 2] it follows that if $\sigma \in \mathbb{T}$ is a regular contact point of an evolution family $\left(\varphi_{s, t}\right)$, then $\sigma(s):=\varphi_{0, s}(\sigma)$ is a regular contact point of the function $\varphi_{s, t}$ for all $t \geq s \geq 0$.

Lemma 3.3. Let $\left(\varphi_{s, t}\right)$ be an evolution family of order $d \in[1,+\infty]$ in $\mathbb{D}$ and let $\sigma \in \mathbb{T}$ be a regular contact point for $\left(\varphi_{s, t}\right)$. Then the spectral function $\Lambda$ of $\left(\varphi_{s, t}\right)$ at $\sigma$ has the following properties:

(i) $\Lambda(0)=0$;

(ii) $\left|\varphi_{s, t}^{\prime}\left(\varphi_{0, s}(\sigma)\right)\right|=e^{\Lambda(s)-\Lambda(t)}$ for any $t \geq s \geq 0$;

(iii) the function $\Lambda$ has locally bounded variation.

Proof. Statement (i) is just by construction, while (ii) follows from the chain rule for angular derivatives, see, e.g., [19, Lemma 2].

Hence we only need to prove (iii). By (ii) and Theorem 2.2 applied to $\varphi=\varphi_{s, t}$ and $z=0$ with $\varphi_{0, s}(\sigma)$ substituted for $\sigma$, for any $t \geq s \geq 0$ we have

$$
[\Lambda(t)-\Lambda(s)]^{+}=\log ^{+} \frac{1}{\left|\varphi_{s, t}^{\prime}\left(\varphi_{0, s}(\sigma)\right)\right|} \leq \log ^{+} \frac{1-\left|\varphi_{s, t}(0)\right|^{2}}{\left|\varphi_{0, t}(\sigma)-\varphi_{s, t}(0)\right|^{2}} \leq \log \frac{1+\left|\varphi_{s, t}(0)\right|}{1-\left|\varphi_{s, t}(0)\right|}
$$

Combined with condition EF3 from Definition 2.12, this inequality implies that the total variation of $\Lambda$ on $[0, T]$ is finite for any $T>0$.

Remark 3.4. In $[8$, Sections 7,8] it has been proved that in case $\tau \in \mathbb{T}$ is the DW-point of $\varphi_{s, t}$ whenever $t \geq s \geq 0$ and $\varphi_{s, t} \neq \mathrm{id}_{\mathbb{D}}$, then the spectral function $\Lambda$ at $\tau$ is absolutely 
continuous and

$$
\Lambda(t)=-\int_{0}^{t} G^{\prime}(\tau, s) d s
$$

Here we study the much more general case of a regular contact point:

Theorem 3.5. Let $\left(\varphi_{s, t}\right)$ be an evolution family and $G$ its Herglotz vector field. Suppose $\left(\varphi_{s, t}\right)$ has a regular contact point $\sigma \in \mathbb{T}$ with spectral function $\Lambda$. Set $\sigma(t):=\varphi_{0, t}(\sigma)$. Then the following statements hold:

(i) for a.e. $t \geq 0$ the angular limit

$$
G(\sigma(t), t):=\angle \lim _{z \rightarrow \sigma(t)} G(z, t)
$$

exists and $v(t):=-i \overline{\sigma(t)} G(\sigma(t), t) \in \mathbb{R} ;$

(ii) the function $[0,+\infty) \ni t \mapsto G(\sigma(t), t)$ is of class $L_{\mathrm{loc}}^{1}$;

(iii) the function $[0,+\infty) \ni t \mapsto \sigma(t)$ is locally absolutely continuous and for any $s, t \geq 0$,

$$
\sigma(t)=\sigma(s)+\int_{s}^{t} G(\sigma(\xi), \xi) d \xi
$$

(iv) for a.e. $t \geq 0$ the angular limit

$$
G^{\prime}(\sigma(t), t):=\angle \lim _{z \rightarrow \sigma(t)} \frac{G(z, t)-G(\sigma(t), t)}{z-\sigma(t)}
$$

exists and $\operatorname{Im} G^{\prime}(\sigma(t), t)=v(t)$;

(v) the function $[0,+\infty) \ni t \mapsto G^{\prime}(\sigma(t), t)$ is of class $L_{\mathrm{loc}}^{1}$ and for any $t \geq 0$,

$$
\Lambda(t)=\int_{0}^{t} \operatorname{Re} G^{\prime}(\sigma(\xi), \xi) d \xi
$$

Remark 3.6. Note that by Theorem 2.2(vi), $\varphi_{s, t}^{\prime}(\sigma(s))=\sigma(t) \overline{\sigma(s)}\left|\varphi_{s, t}^{\prime}(\sigma(s))\right|$ for all $s \geq 0$ and all $t \geq s$. Therefore, statements (iii) - (v) of the above theorem implies that

$$
\varphi_{s, t}^{\prime}(\sigma(s))=\exp \int_{s}^{t} G^{\prime}(\sigma(\xi), \xi) d \xi, \quad \text { for all } t \geq s \geq 0 .
$$

Proof of Theorem 3.5. We divide the proof in several steps.

Step 1. The function $[0,+\infty) \mapsto \sigma(t)$ is of locally bounded variation.

For $t \geq 0$ we denote

$$
h_{t}(z):=\frac{z+\zeta(t)}{1+\overline{\zeta(t)} z},
$$

where $\zeta(t):=\varphi_{0, t}(0)$. Note that $h_{t}$ is an automorphism of $\mathbb{D}$ and $h_{t}(0)=\zeta(t)$ for all $t \geq 0$. For $t \geq s \geq 0$ let $\psi_{s, t}(z):=h_{t}^{-1} \circ \varphi_{s, t} \circ h_{s}$. Clearly $\psi_{s, t} \in \operatorname{Hol}(\mathbb{D}, \mathbb{D}), \psi_{s, t}(0)=0$, and $\psi_{s, t}(b(s))=b(t)$, where $b(t):=h_{t}^{-1}(\sigma(t))$ for all $t \geq 0$. Since the function $t \mapsto \zeta(t) \in \mathbb{D}$ is 
locally absolutely continuous, it is sufficient to prove that $t \mapsto b(t)$ is of locally bounded variation on $[0,+\infty)$. Indeed, for any $t \geq s \geq 0$,

$$
\begin{aligned}
|\sigma(t)-\sigma(s)| & =\left|h_{t}(b(t))-h_{s}(b(s))\right| \leq\left|h_{t}(b(t))-h_{t}(b(s))\right|+\left|h_{t}(b(s))-h_{s}(b(s))\right| \\
& \leq \frac{|b(t)-b(s)|}{1-|\zeta(t)|^{2}}+2 \frac{|\zeta(t)-\zeta(s)|+|\operatorname{lm}(\zeta(t) \overline{\zeta(s)})|}{(1-|\zeta(t)|)(1-|\zeta(s)|)} \\
& \leq \frac{|b(t)-b(s)|}{1-|\zeta(t)|^{2}}+\frac{4|\zeta(t)-\zeta(s)|}{(1-|\zeta(t)|)(1-|\zeta(s)|)} .
\end{aligned}
$$

Fix now $s \geq 0$ and $t \geq s$. Consider $f \in \operatorname{Hol}(\mathbb{D}, \mathbb{D})$ defined as $f(z):=b(s) \overline{b(t)} \psi_{s, t}(z) / z$ for all $z \neq 0$ and $f(0):=b(s) \overline{b(t)} \psi_{s, t}^{\prime}(0)$. By construction, $b(s)$ is a contact point for $f$, $f(b(s))=1$ and the boundary dilation coefficient of $f$ at $b(s)$ is $b(s) \overline{b(t)} \psi_{s, t}^{\prime}(b(s))-1=$ $\left|\psi_{s, t}^{\prime}(b(s))\right|-1>0$ by Theorem 2.2 (vi) and Theorem 2.3. Applying Theorem 2.2 to $f$ at $b(s)$ with $z=0$ we immediately get

$$
\left|1-b(s) \overline{b(t)} \psi_{s, t}^{\prime}(0)\right| \leq 2 \frac{\left|1-b(s) \overline{b(t)} \psi_{s, t}^{\prime}(0)\right|^{2}}{1-\left|\psi_{s, t}^{\prime}(0)\right|^{2}} \leq 2\left(\left|\psi_{s, t}^{\prime}(b(s))\right|-1\right) .
$$

Therefore,

$$
\begin{aligned}
|b(t)-b(s)|=|b(t) / b(s)-1| \leq\left|1-\psi_{s, t}^{\prime}(0)\right| & +\left|\psi_{s, t}^{\prime}(0)-b(t) / b(s)\right| \\
& \leq\left|1-\psi_{s, t}^{\prime}(0)\right|+2\left(\left|\psi_{s, t}^{\prime}(b(s))\right|-1\right) .
\end{aligned}
$$

Note that by [14, Lemma 2.8], $\left(\psi_{s, t}\right)$ is an evolution family. Furthermore, by construction, the origin is the common DW-point of $\left(\psi_{s, t}\right)$ and $b(0)=\sigma$ is a regular contact point of $\left(\psi_{s, t}\right)$. Denote by $\Lambda_{0}$ the spectral function of $\left(\psi_{s, t}\right)$ at $\sigma$. Then (3.1) can be rewritten as

$$
|b(t)-b(s)| \leq\left|1-\psi_{0, t}^{\prime}(0) / \psi_{0, s}^{\prime}(0)\right|+2\left|1-e^{\Lambda_{0}(s)-\Lambda_{0}(t)}\right| \quad \text { for any } t \geq s \geq 0 .
$$

Therefore, the statement of Step 1 follows from the fact that by [8, Theorem 7.1], $[0,+\infty) \ni t \mapsto \psi_{0, t}^{\prime}(0) \neq 0$ is locally absolutely continuous and by Lemma $3.3, \Lambda_{0}$ is of locally bounded variation on $[0,+\infty)$.

Step 2. Assertions (i), (ii) and (iv) hold.

By Lemma 3.3 and the previous step, $\Lambda$ and $t \mapsto \sigma(t)$ are of locally bounded variation on $[0,+\infty)$. Therefore there exists a null-set $N \subset[0,+\infty)$ such that for any $t \in[0,+\infty) \backslash$ $N$, the derivatives $\Lambda^{\prime}(t)$ and $\sigma^{\prime}(t)$ exist finitely and moreover (see, e.g., [16, Theorem 3.6]) for any $s \geq 0$ and $z \in \mathbb{D}$ the map $[s,+\infty) \ni t \mapsto \varphi_{s, t}(z) \in \mathbb{D}$ is differentiable on $[s,+\infty) \backslash N$, with $(\partial / \partial t) \varphi_{s, t}(z)=G\left(\varphi_{s, t}(z), t\right)$ for all such $t$. Consider the family $\left(\tilde{\varphi}_{s, t}\right)$ defined by $\tilde{\varphi}_{s, t}(z)=\overline{\sigma(t)} \varphi_{s, t}(\sigma(s) z)$ for $t \geq s \geq 0$ and $z \in \mathbb{D}$. A priori we cannot state that $\left(\tilde{\varphi}_{s, t}\right)$ is an evolution family. However, for all $s \geq 0$ and all $z \in \mathbb{D}$, the map $[s,+\infty) \ni$ $t \mapsto \tilde{\varphi}_{s, t}(z) \in \operatorname{Hol}(\mathbb{D}, \mathbb{C})$ is differentiable on $[s,+\infty) \backslash N$, with $\frac{\partial}{\partial t} \tilde{\varphi}_{s, t}(z)=\tilde{G}\left(\tilde{\varphi}_{s, t}(z), t\right)$ for 
all $t \in[s,+\infty) \backslash N$, where we set

$$
\tilde{G}(z, t):=\overline{\sigma(t)}\left(G(\sigma(t) z, t)-z \sigma^{\prime}(t)\right)
$$

Note also that $\sigma_{0}=1$ is a BRFP for $\tilde{\varphi}_{s, t}$, and by Theorem 2.2, $\tilde{\varphi}_{s, t}^{\prime}(1)=\left|\varphi_{s, t}^{\prime}(\sigma(s))\right|$ for all $t \geq s \geq 0$.

For any $t_{0} \in[0,+\infty) \backslash N$, the semigroup $\left(\phi_{t}^{t_{0}}\right)$ generated by $\tilde{G}\left(\cdot, t_{0}\right)$ is given by the product formula (see, e.g. [31, Theorem 3])

$$
\phi_{t}^{t_{0}}=\lim _{n \rightarrow+\infty} \phi_{n, t}^{t_{0}}, \quad \text { where } \phi_{n, t}^{t_{0}}:=\left(\tilde{\varphi}_{t_{0}, t_{0}+t / n}\right)^{\circ n}
$$

for all $t \geq 0$. According to the chain rule for angular derivatives, see, e.g., [19, Lemma 2] or $[1$, Lemma (1.3.25) in $\S 1.3 .4], \phi_{n, t}^{t_{0}}$ has a BRFP at 1 for all $t \geq 0, n \in \mathbb{N}$ and

$$
\left(\phi_{n, t}^{t_{0}}\right)^{\prime}(1)=\exp n\left(\Lambda\left(t_{0}\right)-\Lambda\left(t_{0}+t / n\right)\right) .
$$

Then $\left(\phi_{n, t}^{t_{0}}\right)^{\prime}(1) \rightarrow e^{-\Lambda^{\prime}\left(t_{0}\right) t}$ as $n \rightarrow+\infty$. Using Theorem 2.2 (iii) for $\phi_{n, t}^{t_{0}}$ and passing to the limit as $n \rightarrow \infty$, it is not hard to see that 1 is a BRFP for $\phi_{t}^{t_{0}}$ and $\left(\phi_{t}^{t_{0}}\right)^{\prime}(1) \leq e^{-\Lambda^{\prime}\left(t_{0}\right) t}$. Then, by Theorem 2.8, $\tilde{G}\left(\cdot, t_{0}\right)$ has a BRNP at 1 with dilation $\tilde{G}^{\prime}\left(1, t_{0}\right) \leq-\Lambda^{\prime}\left(t_{0}\right)$ for all $t_{0} \in[0,+\infty) \backslash N$.

On the other hand from $(2.3)$ with $\sigma:=1, G:=\tilde{G}\left(\cdot, t_{0}\right), \lambda:=\tilde{G}^{\prime}\left(1, t_{0}\right)$ applied for $z=0$, one easily obtains that $\tilde{G}^{\prime}\left(1, t_{0}\right) \geq-2 \operatorname{Re} \tilde{G}\left(0, t_{0}\right)$ for all $t_{0} \in[0,+\infty) \backslash N$.

Thus we conclude that for any $t \in[0,+\infty) \backslash N$ the infinitesimal generator $\tilde{G}(\cdot, t)$ has a $\mathrm{BRNP}$ at $\sigma_{0}=1$ and

$$
-2 \operatorname{Re} \overline{\sigma(t)} G(0, t) \leq \tilde{G}^{\prime}(1, t) \leq-\Lambda^{\prime}(t) .
$$

Recall also that $\sigma(t) \in \mathbb{T}$ for all $t \in[0,+\infty) \backslash N$. In particular, it follows that assertions (i) and (iv) hold, with $G(\sigma(t), t)=\sigma^{\prime}(t)$,

$$
v(t)=-i \sigma^{\prime}(t) / \sigma(t), \quad \text { and } G^{\prime}(\sigma(t), t)=\tilde{G}^{\prime}(1, t)+i v(t) \quad \text { for all } t \in[0,+\infty) \backslash N
$$

Finally, assertion (ii) also holds because $t \mapsto \sigma^{\prime}(t)$ is locally integrable on $[0,+\infty)$.

Step 3. Assertion (iii) holds.

Recall that the angular derivative coincides, provided it is finite, with the radial limit of the derivative (see, e.g., [29, Prop. 4.7 on p. 79]). Therefore, by (3.5), we have

$$
\tilde{G}^{\prime}(1, t)+i v(t)=G^{\prime}(\sigma(t), t)=\lim _{(0,1) \ni x \rightarrow 1} G^{\prime}(x \sigma(t), t) \quad \text { for any } t \in[0,+\infty) \backslash N .
$$

Since $t \mapsto \sigma(t)$ is continuous, from conditions H1 and H2 in Definition 2.11 it follows that $t \mapsto G^{\prime}(x \sigma(t), t)$ is measurable on $[0,+\infty)$ for all $x \in(0,1)$. Therefore, $t \mapsto G^{\prime}(\sigma(t), t)$ is measurable too. Now, since $t \mapsto v(t)$ is locally integrable, (3.4)-(3.5) implies that $t \mapsto G^{\prime}(\sigma(t), t)$ is also locally integrable on $[0,+\infty)$. 
Fix now any $t>0$ and write

$$
\varphi_{0, t}(x \sigma)=x \sigma+\int_{0}^{t} G\left(\varphi_{0, s}(x \sigma), s\right) d s .
$$

Since the spectral function $\Lambda$ has finite variation, it follows that there exists $M_{t}>0$ such that $\left|\varphi_{0, s}^{\prime}(\sigma)\right|<M_{t}$ for al $s \in[0, t]$. By Theorem 2.2 applied to $\varphi_{0, s}$ and $z=x \sigma$,

$$
\frac{\left|\sigma(s)-\varphi_{0, s}(x \sigma)\right|^{2}}{1-\left|\varphi_{0, s}(x \sigma)\right|^{2}} \leq M_{t} \frac{1-x}{1+x} \quad \text { for all } x \in(0,1) \text { and all } s \in[0, t] .
$$

According to Lebesgue's Dominated Convergence Theorem, combining assertion (i), Lemma 2.10 applied to $\tilde{G}(\cdot, t), t \in[0,+\infty) \backslash N$, inequality (3.7), and the fact that the functions $t \mapsto G^{\prime}(\sigma(t), t), t \mapsto G(0, t)$, and $t \mapsto v(t)$ are locally integrable on $[0,+\infty)$, we obtain (iii) by passing to the limit in $(3.6)$ as $(0,1) \ni x \rightarrow 1$.

Step 4. Assertion (v) holds.

According to the previous step of the proof, $t \mapsto \sigma(t)$ is locally absolutely continuous on $[0,+\infty)$. Therefore, by [14, Lemma 2.8], $\left(\tilde{\varphi}_{s, t}\right)$ is an evolution family. By construction, $\tilde{G}$ is its Herglotz vector field, $\sigma_{0}=1$ is its RBFP, and $\Lambda$ is its spectral function at $\sigma_{0}$. Therefore, bearing in mind (3.5), in order to prove (v) we may assume that $\sigma(t) \equiv 1$.

From (3.6), fixing any $t \geq 0$ we can write

$$
e^{-\Lambda(t)}=\varphi_{0, t}^{\prime}(1)=\lim _{\substack{x \rightarrow 1 \\ x \in(0,1)}} \frac{1-\varphi_{0, t}(x)}{1-x}=1+\lim _{\substack{x \rightarrow 1 \\ x \in(0,1)}} \int_{0}^{t} \frac{G\left(\varphi_{0, s}(x), s\right)}{x-1} d s .
$$

Arguing as at the end of Step 3, use (3.7) and Lemma 2.10 to conclude that the integrand in (3.8) does not exceed in absolute value $C_{t}\left(|G(0, s)|+\left|G^{\prime}(1, s)\right| / 2\right)$ for all $s \in[0, t] \backslash N$, all $x \in(0,1)$, and some constant $C_{t}>0$ not depending on $s$ and $x$. Recalling that $G(0, \cdot)$ and $G^{\prime}(1, \cdot)$ are locally integrable, we can pass to the limit in (3.8) using Lebesgue's Dominated Convergence Theorem. Hence we obtain

$$
e^{-\Lambda(t)}=1+\int_{0}^{t} G^{\prime}(1, s) \varphi_{0, s}^{\prime}(1) d s \quad \text { for all } t \geq 0 .
$$

Differentiating (3.9) w.r.t. $t$, we get $-\Lambda^{\prime}(t) e^{-\Lambda(t)}=G^{\prime}(1, t) \varphi_{0, t}^{\prime}(1)=G^{\prime}(1, t) e^{-\Lambda(t)}$ for a.e. $t \geq 0$, from which $(\mathrm{v})$ follows easily. The proof is now complete.

\section{The PRoOF OF TheOREM 1.1}

In this section we are going to prove Theorem 1.1. We will make use the following lemma, whose proof follows almost literally an argument in the proof of $[29$, Theorem 10.5, p. 305-306], so we omit it.

Lemma 4.1. Let $g: \mathbb{D} \rightarrow \mathbb{C}$ be a univalent holomorphic function, $\sigma \in \mathbb{T}$, and $\omega \in \mathbb{C} \backslash g(\mathbb{D})$. Then the function $\psi(z):=(g(z)-\omega) /(z-\sigma)$ is normal in $\mathbb{D}$. In particular, if $C$ is a slit in $\mathbb{D}$ landing at $\sigma$ and the limit $L:=\lim _{C \ni z \rightarrow \sigma} \psi(z)$ exists, then the angular limit of $\psi$ at $\sigma$ 
also exists and equals $L$. If in addition $L \neq \infty$, then $\angle \lim _{z \rightarrow \sigma} g(z)=\omega$ and the angular derivative $g^{\prime}(\sigma)$ of $g$ at $\sigma$ exists and equals $L$.

We can now start proving Theorem 1.1. Without loss of generality we assume that $\sigma=1$. Note that if (A) holds, then (B) and (1.1) follow directly from Theorem 3.5.

Let us show that (B) implies (A). Let $u(z):=-\left(1-|z|^{2}\right) /|1-z|^{2}$ be the (negative) Poisson kernel and define

$$
g_{z}(s, t):=u\left(\varphi_{s, t}(z)\right)-e^{\Lambda_{0}(t)-\Lambda_{0}(s)} u(z), \quad \Lambda_{0}(t):=-\int_{0}^{t} G^{\prime}(1, \xi) d \xi,
$$

for $z \in \mathbb{D}$ and $t \geq s \geq 0$. By Theorem 2.2 (see also [10, Proposition 2.3]) the inequality

$$
g_{z}(s, t) \leq 0, \quad \forall z \in \mathbb{D} \forall 0 \leq s \leq t,
$$

is equivalent to $\varphi_{s, t}$ having a BRFP at 1 with $\varphi_{s, t}^{\prime}(1) \leq e^{\Lambda_{0}(s)-\Lambda_{0}(t)}$.

Since by (B) for a.e. $t \geq 0$ the infinitesimal generator $G(\cdot, t)$ has a BRNP of dilation $G^{\prime}(1, t)$ at $\sigma=1$, it follows from [10, Theorem 0.4] that for all $z \in \mathbb{D}$ and a.e. $t \geq 0$,

$$
\operatorname{Re}(v(z) G(z, t))+G^{\prime}(1, t) u(z) \leq 0, \quad \text { where } v:=\partial u / \partial x-i \partial u / \partial y .
$$

Fix now $z \in \mathbb{D}$ and $s \geq 0$. Taking into account that $t \mapsto \varphi_{s, t}(z)$ solves the equation $(\partial / \partial t) \varphi_{s, t}(z)=G\left(\varphi_{s, t}(z), t\right)$ on $[s,+\infty)$, we conclude that $t \mapsto g_{z}(s, t)$ is locally absolutely continuous on $[s,+\infty)$ and, with the notation $w(t):=\varphi_{s, t}(z)$,

$$
\frac{\partial g_{z}(s, t)}{\partial t}=\operatorname{Re}(v(w(t)) G(w(t), t))+G^{\prime}(1, t) u(w(t))-G^{\prime}(1, t) g_{z}(s, t) \leq-G^{\prime}(1, t) g_{z}(s, t)
$$

for a.e. $t \geq s$. Therefore, $h(t):=-(\partial / \partial t) g_{z}(s, t)-G^{\prime}(1, t) g_{z}(s, t) \geq 0$ for a.e. $t \geq s$. Note that $t \mapsto g_{z}(s, t)$ is the solution to the differential equation $(\partial / \partial t) g_{z}(s, t)+G^{\prime}(1, t) g_{z}(s, t)+$ $h(t)=0$ with the initial condition $g_{z}(s, s)=0$. Thus one easily concludes that $g_{z}(s, t) \leq 0$ for all $t \geq s$. This proves the implication $(\mathrm{B}) \Longrightarrow(\mathrm{A})$.

The proof of the equivalence between $(\mathrm{A})$ and $(\mathrm{C})$ is divided into several steps.

Step 1. (C.1) and (C.2) imply that for each $t \geq s \geq 0$ the point 1 is a contact point of $\varphi_{s, t}$. Moreover, if for some $t \geq s \geq 0, \varphi_{s, t}(1) \neq 1$, then $\arg \left(f_{t}^{\prime}(1) / f_{s}^{\prime}(1)\right)=\pi$.

Fix any $t \geq s \geq 0$. Since by hypothesis $\lim _{r \rightarrow 1} f_{s}(r)=f_{t_{0}}(1) \notin f_{t}(\mathbb{D})$, by $[22$, Theorem 1 , $\S$ II.3] the univalence of $f_{t}$ implies that the $\operatorname{limit}_{\lim } \lim _{r \rightarrow 1}(r)=\lim _{r \rightarrow 1}\left(f_{t}^{-1} \circ f_{s}\right)(r)$ does exist and belongs to $\mathbb{T}$. Thus 1 is a contact point of $\varphi_{s, t}$.

Let us now assume that $\varphi_{s, t}(1) \neq 1$. Since $f_{t}$ is conformal at 1 , it is also isogonal at this point (here we follow the terminology from $[30, \S 4.3]$ ). Therefore (see, e.g., [30, Theorem 11.6]) we have:

(a) for each $\alpha \in(0, \pi / 2)$ there exists $\rho_{1}>0$ such that

$$
S\left(\alpha, \rho_{1}, \kappa_{t}\right):=\left\{w \in \mathbb{C}:\left|\arg \bar{\kappa}_{t}(w-p)\right|<\alpha,|w-p|<\rho_{1}\right\} \subset f_{t}(\mathbb{D}),
$$

where $\kappa_{t}:=-f_{t}^{\prime}(1) /\left|f_{t}^{\prime}(1)\right|$ and $p:=f_{t}(1)=f_{t_{0}}(1)$; 
(b) for each $\alpha>\pi / 2$ there exist $\underline{n o} \kappa \in \mathbb{T}$ and $\rho>0$ such that $S(\alpha, \rho, \kappa) \subset f_{t}(\mathbb{D})$. Similarly,

(c) $S\left(\alpha, \kappa_{s}, \rho_{2}\right) \subset f_{s}(\mathbb{D}) \subset f_{t}(\mathbb{D})$ for all $\alpha \in(0, \pi / 2)$ and some $\rho_{2}>0$ depending on $\alpha$.

It follows that $\theta:=\arg \left(f_{t}^{\prime}(1) / f_{s}^{\prime}(1)\right) \in\{0, \pi\}$. Indeed, if $\theta \in(-\pi, 0) \cup(0, \pi)$, then setting $\alpha>\max \{|\theta| / 2, \pi / 2-|\theta| / 2\}$ in (a) and (c) one easily concludes that

$$
S\left(\alpha+|\theta| / 2, \kappa_{s} e^{i \theta / 2}, \rho_{3}\right) \subset f_{t}(\mathbb{D}), \quad \text { where } \rho_{3}:=\min \left\{\rho_{1}, \rho_{2}\right\},
$$

which contradicts (b). Hence $\theta \in\{0, \pi\}$

It remains to show that actually $\theta=\pi$. Suppose on the contrary that $\theta=0$. Then the $\operatorname{arcs} \gamma_{1}:=f_{t}([0,1))$ and $\gamma_{2}:=f_{s}([0,1))$ approach the point $p=f_{t}(\mathbb{D})$ through the same disc sector $S\left(\pi / 4, \rho_{1}, \kappa_{t}\right)$, which is a subset of $f_{t}(\mathbb{D})$ for $\rho_{1}>0$ small enough. Hence $\gamma_{1}$ and $\gamma_{2}$ are equivalent as slits in $f_{t}(\mathbb{D})$, i.e. they represent the same accessible boundary point of $f_{t}(\mathbb{D})$. Then by [22, Theorem 1, §II.3], $[0,1)=f_{t}^{-1}\left(\gamma_{1}\right)$ and $\varphi_{s, t}([0,1))=f_{t}^{-1}\left(\gamma_{2}\right)$ land at the same point on $\mathbb{T}$, namely at the point 1 . It follows that $\varphi_{s, t}(1)=1$, which contradicts our assumption. Thus $\theta=\pi$.

Step 2. If $(C)$ holds, then $(A)$ holds too and $t \mapsto f_{t}^{\prime}(\sigma)$ is locally absolutely continuous on $[0,+\infty)$, with $\arg f_{t}^{\prime}(\sigma)$ being constant.

We are going to prove that 1 is a regular fixed point for all $\varphi_{s, t}$ 's. By Step 1 , the point 1 is a contact point of $\varphi_{s, t}$ for any $t \geq s \geq 0$. Let us now fix $s \geq 0$ and study the map

$$
\Phi_{s}:[s,+\infty) \ni t \mapsto \varphi_{s, t}(1) \in \mathbb{T} .
$$

Step 2.1. The map $\Phi_{s}$ is continuous.

Suppose on the contrary that there exists $\varepsilon_{0}>0$, a point $t_{0} \geq s$ and a convergent sequence $[s,+\infty) \ni t_{n} \rightarrow t_{0}$ such that $\left|\varphi_{s, t_{n}}(1)-\varphi_{s, t_{0}}(1)\right|>\varepsilon_{0}$. From the fact (see [8, Proposition 3.5]) that $\varphi_{s, t_{n}} \rightarrow \varphi_{s, t_{0}}$ locally uniformly in $\mathbb{D}$ as $n \rightarrow+\infty$ it follows that passing if necessary to a subsequence of $\left(t_{n}\right)$ we may assume that $\left|\varphi_{s, t_{n}}\left(r_{n}\right)-\varphi_{s, t_{0}}(1)\right|<$ $\varepsilon_{0} / 2$, where $r_{n}:=1-1 / n$, for all $n \in \mathbb{N}$. Now fix any $T>s$ such that $\left(t_{n}\right) \subset[s, T]$. The sets $C_{n}:=\varphi_{s, t_{n}}\left(\left[r_{n}, 1\right)\right) \subset \mathbb{D}$ form a sequence of Kobe arcs for the sequence of functions $\varphi_{t_{n}, T}$. Indeed, on the one hand by construction, $\operatorname{diam}_{\mathbb{C}}\left(C_{n}\right)>\varepsilon_{0} / 2$ for all $n \in \mathbb{N}$; while on the other hand, $\varphi_{t_{n}, T}\left(C_{n}\right)=\varphi_{s, T}\left(\left[r_{n}, 1\right)\right)$ tends, as $n \rightarrow+\infty$, to the point $w_{0}:=\varphi_{s, T}(1)$. By the Schwarz - Pick theorem, $\left|\varphi_{t_{n}, T}^{\prime}(z)\right|\left(1-|z|^{2}\right) \leq 1$ for all $z \in \mathbb{D}$ and all $n \in \mathbb{N}$. Hence by [29, Theorem 9.2, p. 265], $\varphi_{t_{n}, T}-w_{0} \rightarrow 0$ as $n \rightarrow+\infty$. However, by [8, Proposition 3.5], $\left(\varphi_{t_{n}, T}\right)$ converges to $\varphi_{t_{0}, T}$ which is univalent in $\mathbb{D}$ (see [8, Corollary 6.3]). This contradiction proves the statement of Step 2.1.

Step 2.2. There exists $\varepsilon>0$ such that $\varphi_{s, t}(1)=1$ for all $t \in[s, s+\varepsilon)$.

According to (C.3) we can choose $\varepsilon>0$ so that $\left|\arg \left(f_{t}^{\prime}(1) / f_{s}^{\prime}(1)\right)\right|<\pi$ for all $t \in[s, s+\varepsilon)$. Applying Step 1, we easily conclude that $\varphi_{s, t}(1)=1$ for all such $t$.

Step 2.3. Fix $t \geq s$ and suppose that $\varphi_{s, t}(1)=1$. Then there exists the finite angular derivative $\varphi_{s, t}^{\prime}(1)=f_{s}^{\prime}(1) / f_{t}^{\prime}(1)>0$. 
By [30, Proposition 4.13], there exists the angular derivative $\varphi_{s, t}^{\prime}(1)$, which can be either $\infty$ or a positive number. Therefore,

$$
\frac{f_{t}\left(\varphi_{s, t}(r)\right)-f_{t}(1)}{\varphi_{s, t}(r)-1}=\frac{f_{s}(r)-f_{s}(1)}{r-1} \frac{r-1}{\varphi_{s, t}(r)-1} \rightarrow f_{s}^{\prime}(1) \frac{1}{\varphi_{s, t}^{\prime}(1)} \in \mathbb{C}
$$

as $(0,1) \ni r \rightarrow 1$. Thus using Lemma 4.1 for $g:=f_{t}, \sigma:=1, \omega:=f_{t}(1) \notin f_{t}(\mathbb{D})$, and $C:=\varphi_{s, t}([0,1))$, we conclude that $\varphi_{s, t}^{\prime}(1)=f_{s}^{\prime}(1) / f_{t}^{\prime}(1) \neq \infty$, which proves the statement of Step 2.3.

Step 2.4. For any $t \geq s, \varphi_{s, t}(1)=1$.

Suppose this is not the case. Let $t_{*}:=\inf \left\{t \geq s: \varphi_{s, t}(1) \neq 1\right\}$. Then, by Step 2.2, $t_{*}>s$ and for all $t \in\left(s, t_{*}\right)$ we have $\varphi_{s, t}(1)=1$. Hence by Step 2.1, $\varphi_{s, t_{*}}(1)=1$. Furthermore, by Step 2.3 applied to $t:=t_{*}, \varphi_{s, t_{*}}^{\prime}(1) \in \mathbb{C}$. Therefore, $\varphi_{s, t}(1)=\varphi_{t_{*}, t}(1)$ for all $t \geq t_{*}$. But by Step 2.2 applied with $t_{*}$ substituted for $s, \varphi_{t_{*}, t}(1)=1$ provided $t-t_{*}$ is small enough. Thus $t_{*}<\inf \left\{t \geq s: \varphi_{s, t}(1) \neq 1\right\}$. This contradiction proves the statement of Step 2.4.

Statements of Step 2.3 and 2.4 imply assertion (A). Hence (1.1) holds and, in particular, $t \mapsto f_{t}^{\prime}(1)=f_{0}^{\prime}(1) e^{\Lambda(t)}$ is locally absolutely continuous. The proof of Step 2 is complete.

Step 3. (A) implies $(C)$.

For any $s \geq 0$ and $t \geq s$, since the point 1 is a BRFP of the function $\varphi_{s, t}$, the angular derivative $\varphi_{s, t}^{\prime}(1)$ is a positive number (see, e.g., [30, Proposition 4.13]).

Fix $t \geq 0$. Assume first that $t>t_{0}$. Then $f_{t}\left(\varphi_{t_{0}, t}(r)\right)=f_{t_{0}}(r)$ for all $r \in[0,1)$. In particular, $f_{t_{0}}(1) \in \partial f_{t}(\mathbb{D})$, because $\varphi_{t_{0}, t}(r) \rightarrow 1$ as $(0,1) \ni r \rightarrow 1$. Moreover,

$$
\frac{f_{t}\left(\varphi_{t_{0}, t}(r)\right)-f_{t_{0}}(1)}{\varphi_{t_{0}, t}(r)-1}=\frac{f_{t_{0}}(r)-f_{t_{0}}(1)}{r-1} \frac{r-1}{\varphi_{t_{0}, t}(r)-1} \rightarrow f_{t_{0}}^{\prime}(1) \frac{1}{\varphi_{t_{0}, t}^{\prime}(1)} \in \mathbb{C}^{*}
$$

as $(0,1) \ni r \rightarrow 1$. Therefore, by Lemma 4.1 applied to $g:=f_{t}, \sigma:=1, \omega:=f_{t_{0}}(1) \notin f_{t}(\mathbb{D})$, and $C:=\varphi_{s, t}([0,1))$, the angular $\operatorname{limit}_{\lim _{z \rightarrow 1}} f_{t}(z)$ exists and equals $f_{t_{0}}(1)$, and the angular derivative $f_{t}^{\prime}(1)$ of $f_{t}$ at 1 exists and equals $f_{t_{0}}^{\prime}(1) / \varphi_{t_{0}, t}^{\prime}(1) \in \mathbb{C}^{*}$.

Now consider the case $t<t_{0}$. We have $f_{t}(\mathbb{D}) \subset f_{t_{0}}(\mathbb{D}) \not \supset f_{t_{0}}(1)$. Note that the curve $r \in[0,1) \mapsto \varphi_{t, t_{0}}([0,1))$ approaches the point 1 tangentially to the real axis. Therefore,

$$
\frac{f_{t}(r)-f_{t_{0}}(1)}{r-1}=\frac{f_{t_{0}}\left(\varphi_{t, t_{0}}(r)\right)-f_{t_{0}}(1)}{\varphi_{t, t_{0}}(r)-1} \frac{\varphi_{t, t_{0}}(r)-1}{r-1} \rightarrow f_{t_{0}}^{\prime}(1) \varphi_{t, t_{0}}^{\prime}(1) \in \mathbb{C}^{*}
$$

as $(0,1) \ni r \rightarrow 1$. Again using Lemma 4.1 for $g:=f_{t}, \sigma:=1, \omega:=f_{t_{0}}(1)$, and $C:=[0,1)$, we conclude that the angular $\operatorname{limit} \angle \lim _{z \rightarrow 1} f_{t}(z)$ exists and equals $f_{t_{0}}(1)$ and that the angular derivative $f_{t}^{\prime}(1)$ of $f_{t}$ at 1 exists and equals $f_{t_{0}}^{\prime}(1) \varphi_{t, t_{0}}^{\prime}(1) \in \mathbb{C}^{*}$.

Note that in both cases $f_{t}^{\prime}(1) / f_{t_{0}}^{\prime}(1)>0$. Thus the proof is now complete.

\section{Embedding into EVOLUtion FAMiLIES AND INCLUSION CHAinS}

In this section we prove Theorem 1.2. Let us recall the following definition from [15]: 
Definition 5.1. Let $D_{1} \subset D_{2} \subsetneq \mathbb{C}$ be two simply connected domains, let $F_{j}$, for $j=1,2$, be a conformal mapping of $\mathbb{D}$ onto $D_{j}$ and let $P$ be a prime end of the domain $D_{1}$. The domain $D_{1}$ is said to be conformally embedded in the domain $D_{2}$ at the prime end $P$ if there exists a regular contact point $\xi \in \mathbb{T}$ of the mapping $\varphi:=F_{2}^{-1} \circ F_{1}$ such that the point $\omega:=\varphi(\xi)$ corresponds under the mapping $F_{1}$ to the prime end $P$.

For the definition of primes ends and their correspondence under conformal mappings, see e.g. $\left[12\right.$, Chapter 9]. Note that the choice of the conformal mappings $F_{j}$ in the above definition is clearly irrelevant.

Denote by $r\left(w_{0}, D\right)$ the conformal radius of a simply connected domain $D$ w.r.t. a point $w_{0} \in D$. First we prove the following statement:

Proposition 5.2. Let $\left(D_{t} \subsetneq \mathbb{C}\right)_{t \geq 0}$ be a family of simply connected domains and $\Lambda:[0,+\infty) \rightarrow \mathbb{R}$ a locally absolutely continuous function with $\Lambda(0)=0$. The following three statements are equivalent:

(I) The family $\left(D_{t}\right)$ satisfies the following conditions:

(Ia) $D_{s} \subset D_{t}$ whenever $0 \leq s \leq t<+\infty$;

(Ib) the function $[0,+\infty) \ni t \mapsto r\left(w_{0}, D_{t}\right) \in(0,+\infty)$ is locally absolutely continuous for some (and hence every) point $w_{0} \in D_{0}$;

(Ic) there exists a prime end $P$ of the domain $D_{0}$ such that for each $t>0$ the domain $D_{0}$ is embedded in $D_{t}$ conformally at the prime end $P$.

(II) There exists a Loewner chain $\left(f_{t}\right)$ that satisfies the following conditions:

(IIa) $f_{t}(\mathbb{D})=D_{t}$ for all $t \geq 0$;

(IIb) the evolution family $\varphi_{s, t}:=f_{t}^{-1} \circ f_{s}, 0 \leq s \leq t<+\infty$, of $\left(f_{t}\right)$ has a boundary regular fixed point at 1 ;

(IIc) the spectral function of $\left(\varphi_{s, t}\right)$ at the point 1 coincides with $\Lambda$.

(III) There exists a Loewner chain $\left(f_{t}\right)$ that satisfies the above conditions (IIa) and (IIb) (but not necessarily (IIC)).

Remark 5.3. By [15, Theorem 1.8 and Remark 1.7], conditions (Ia) and (Ib) imply that: $(\mathrm{KC} 1)$ for any $t>0, D_{t}=\bigcup_{s \in[0, t)} D_{s}$;

(KC2) for any $s \geq 0, D_{s}$ is a connected component of the interior of the set $\bigcap_{t>s} D_{t}$.

Definition $5.4([15])$. A family $\left(D_{t} \subsetneq \mathbb{C}\right)_{t \geq 0}$ of simply connected domains is said to be:

- an inclusion chain, if it satisfies conditions (Ia), (KC1) and (KC2);

- an L-admissible family if it satisfies conditions (Ia) and (Ib);

- a chordally admissible family, if it satisfies conditions (Ia), (Ib) and (Ic).

Remark 5.5. A result similar to the above proposition, [15, Theorem 4.8], was earlier proved for the case of the common boundary Denjoy-Wolff point. In Proposition 5.2 we consider the more general case of common BRFPs, but even so most of the arguments from [15] can be still used. For example, the equivalence between (II) and (III) 
in Proposition 5.2 is essentially the same as the equivalence between (c) and (d) in [15, Theorem 4.8]. The substantial improvement, made by combining methods from [15] with Theorem 3.5, resides in the fact that, according to Proposition 5.2, for any chordally admissible family $\left(D_{t}\right)$ there exists a Loewner chain $\left(f_{t}\right)$ of chordal type such that $f_{t}(\mathbb{D})=D_{t}$ for all $t \geq 0$, while [15, Theorem 4.8] guarantee only the equality of sets $\left\{f_{t}(\mathbb{D}): t \geq 0\right\}$ and $\left\{D_{t}: t \geq 0\right\}$. The necessity to use Theorem 3.5 in the proof explains why the parameter $d$ (the order of the evolution family $\left(\varphi_{s, t}\right)$ ) does not appear in Proposition 5.2.

Proof of Proposition 5.2. The proof is divided into three steps.

Step 1. $(I I) \Rightarrow(I I I) \Rightarrow(I)$.

The fact that (II) implies (III) is completely trivial. Assume that (III) takes place. Then by [15, Theorem 2.3], conditions (Ia) and (Ib) hold, i.e. $\left(D_{t}\right)$ is an L-admissible family. Now applying $\left[15\right.$, Lemma 4.7] to $\left(F_{t}\right):=\left(f_{t}\right)$ we see that $\left(D_{t}\right)$ is in fact chordally admissible, i.e., (Ic) holds as well.

Step 2. (I) implies (III), with the evolution family $\left(\varphi_{s, t}\right)$ satisfying $\varphi_{s, t}^{\prime}(1)=1$.

By [15, Lemma 4.7] there exists a family of conformal maps $\left(F_{t}: \mathbb{D} \rightarrow \mathbb{C}\right)_{t \geq 0}$ such that $F_{t}(\mathbb{D})=D_{t}$ for all $t \geq 0$ and that the function $\psi_{t}:=F_{t}^{-1} \circ F_{0}$ has a BRFP at 1 . At the same time, by $\left[15\right.$, Theorem 2.3] there exists a Loewner chain $\left(g_{t}\right)$ such that $g_{t}(\mathbb{D})=\Omega_{t}$ for all $t \geq 0$.

Since $g_{t}(\mathbb{D})=F_{t}(\mathbb{D})$, it follows that for any $t \geq 0$ there exists $h_{t} \in \operatorname{Möb}(\mathbb{D})$ such that $F_{t}=g_{t} \circ h_{t}$ and hence $\phi_{0, t}=h_{t} \circ \psi_{t} \circ h_{0}^{-1}$ for all $t \geq 0$, where $\left(\phi_{s, t}\right)$ is the evolution family of the Loewner chain $\left(g_{t}\right)$. It follows that $\sigma_{0}:=h_{0}(1)$ is a regular contact point of $\left(\phi_{s, t}\right)$ and that $h_{t}(1)=\phi_{0, t}\left(\sigma_{0}\right),\left|h_{t}^{\prime}(1)\right| \exp \Lambda_{0}(t)=\left|h_{0}^{\prime}(1)\right|$ for all $t \geq 0$, where $\Lambda_{0}$ is the spectral function of $\left(\phi_{s, t}\right)$ at $\sigma_{0}$. Therefore, there exists a family $\left(\ell_{t}\right)_{t \geq 0} \subset \mathrm{Möb}(\mathbb{D})$ of automorphisms with parabolic fixed point at 1 such that

$$
\tilde{h}_{t}:=h_{t} \circ \ell_{t}=\left(z \mapsto \phi_{0, t}\left(\sigma_{0}\right) \bar{\sigma}_{0} z\right) \circ h_{0} \circ\left(z \mapsto \frac{z+x(t)}{1+x(t) z}\right),
$$

where $x(t):=\left(e^{\Lambda_{0}(t)}-1\right) /\left(e^{\Lambda_{0}(t)}+1\right)$. Note that $\ell_{0}=\mathrm{id}{ }_{\mathbb{D}}$.

By Theorem 3.5, the functions $\Lambda_{0}$ and $t \mapsto \phi_{0, t}\left(\sigma_{0}\right)$ are locally absolutely continuous. Therefore, by [14, Lemma 2.8], the formula $\varphi_{s, t}=\tilde{h}_{t}^{-1} \circ \phi_{s, t} \circ \tilde{h}_{s}, t \geq s \geq 0$, defines an evolution family $\left(\varphi_{s, t}\right)$. Note that the univalent functions $f_{t}:=F_{t} \circ \ell_{t}=g_{t} \circ \tilde{h}_{t}$ satisfy $f_{t} \circ \varphi_{s, t}=f_{s}$ for any $t \geq s \geq 0$. Thus $\left(f_{t}\right)$ is a Loewner chain associated with $\left(\varphi_{s, t}\right)$, see $\left[14\right.$, Lemma 3.2]. Finally, note that $\varphi_{0, t}=\ell_{t}^{-1} \circ \psi_{t}$ has a parabolic fixed point at 1 .

Step 3. $(I) \Rightarrow(I I)$.

We are going to modify the Loewner chain $\left(f_{t}\right)$ constructed in the proof of Step 2 in such a way that the spectral function of the evolution family of this new Loewner chain at 1 coincides with $\Lambda$. To this end we define $m_{t}(z):=(z+x(t)) /(1+x(t) z)$ for all $z \in \mathbb{D}$ and $t \geq 0$, where $x(t):=\left(e^{\Lambda(t)}-1\right) /\left(e^{\Lambda(t)}+1\right)$. Then $\psi_{s, t}:=m_{t} \circ \varphi_{s, t} \circ m_{s}^{-1}$ satisfies $\psi_{s, t}(1)=1$ and $\psi_{s, t}^{\prime}(1)=\exp (\Lambda(s)-\Lambda(t))$ for all $t \geq s \geq 0$. Recall that $\Lambda$ is locally absolutely 
continuous by the hypothesis. Therefore, applying [14, Lemmas 2.8 and 3.2] in the same way as in the proof of Step 2, we see that the functions $g_{t}=f_{t} \circ m_{t}^{-1}$ form a Loewner chain whose evolution family is $\left(\psi_{s, t}\right)$. This is the desired Loewner chain and the proof is now complete.

Remark 5.6. The Loewner chains $\left(f_{t}\right)$ we construct in the proof of Proposition 5.2 are such that the point 1 corresponds under the conformal map $f_{0}: \mathbb{D} \rightarrow D_{0}$ to the prime end $P$ from condition (Ic).

As a preparation to the proof of Theorem 1.2, we need the following lemmata.

Lemma 5.7. Let $G \subsetneq \mathbb{D}$ be a simply connected domain, $w_{0} \in G$, and $t_{0}>0$. Then there exists an L-admissible family of domains $\left(D_{t}\right)$ such that $D_{0}=G, D_{t_{0}}=\mathbb{D}$, and $D_{t} \subset\{z: \operatorname{Re} z<1\}$ for all $t \geq 0$.

Proof. First of all without loss of generality we may assume that $t_{0}=1$. Furthermore, using Möbius transformations we may assume that $w_{0}=0$. Indeed, if $\ell \in \operatorname{Möb}(\mathbb{D})$ and $\left(D_{t}\right)$ is an L-admissible family such that $D_{1}=\mathbb{D}$, then $\left(\tilde{D}_{t}\right)$ defined by $\tilde{D}_{t}:=\ell\left(D_{t}\right)$ for $t \in[0,1]$ and $\tilde{D}_{t}:=D_{t}$ for all $t \geq 1$, is again an L-admissible family.

Denote $M:=1 / r(G, 0)>1$, and let $\phi$ be the conformal map of $\mathbb{D}$ onto $G$ normalized by $\phi(0)=0$ and $\phi^{\prime}(0)>0$. Then the function $f_{0}=M \varphi$ belongs to the class

$$
\mathcal{S}^{M}:=\left\{f(z)=z+\sum_{n=2}^{+\infty} a_{n} z^{n}:|f(z)|<M \text { for all } z \in \mathbb{D} \text { and } f \text { is univalent in } \mathbb{D}\right\} .
$$

It is known (see, e.g., [4, p. 69-70]) that for any $f_{0} \in \mathcal{S}^{M}$ there exists a function $p: \mathbb{D} \times[0, T] \rightarrow \mathbb{C}$, where $T:=\log M$, such that

(i) for any $t \in[0, T]$ the function $p(\cdot, t)$ belongs to the Carathéodory class, i.e. it is a holomorphic function in $\mathbb{D}$ with positive real part and normalized by $p(0, t)=1$;

(ii) for each $z \in \mathbb{D}$ the function $p(z, \cdot)$ is measurable in $[0, T]$;

(iii) for all $z \in \mathbb{D}$ we have $f_{0}(z)=M \psi_{z, 0}(T)$, where $w(t)=\psi_{z, s}(t), s \in[0, T], z \in \mathbb{D}$, stands for the unique solution to the initial value problem

$$
\frac{d w(t)}{d t}=-w(t) p(w(t), t), \quad w(s)=z .
$$

For each $s, t \in[0, T]$ with $s \leq t$ the function $\varphi_{s, t}(z):=\psi_{z, s}(t)$ is a well-defined univalent holomorphic self-map of $\mathbb{D}$ with $\varphi_{s, t}(0)=0, \varphi_{s, t}^{\prime}(0)=e^{s-t}$ and $\varphi_{u, t} \circ \varphi_{s, u}=\varphi_{s, t}$ whenever $0 \leq s \leq u \leq t \leq T$, see, e.g., [29, proof of Theorem 6.3, p. 160-162]. Note also that by construction, $\varphi_{0, T}=\phi$. In particular, it follows that the family of simply connected domains $D_{t}:=\varphi_{(1-t) T, T}(\mathbb{D})$ for all $t \in[0,1]$ and $D_{t}:=t \mathbb{D}+1-t$ for all $t \geq 1$, satisfies conditions $D_{0}=G, D_{1}=\mathbb{D}, D_{s} \subset D_{t}$ whenever $0 \leq s \leq t \leq 1$, and that $0 \leq t \mapsto r\left(D_{t}, 0\right)$ is a piecewise smooth function. Then by the very definition, $\left(D_{t}\right)$ is an L-admissible family. Finally, by construction, $D_{t} \subset\{z: \operatorname{Re} z<1\}$ for all $t \geq 0$. The proof is now complete. 
Remark 5.8. An alternative proof of the above lemma, not using representation of the class $\mathcal{S}^{M}$, can be obtained by a modification of the proof of [29, Theorem 6.1 on p. 159].

Lemma 5.9. Let $D_{0} \subset D_{\infty} \subsetneq \mathbb{C}$ be two simply connected domains. If $D_{0}$ is embedded in $D_{\infty}$ conformally at some prime end $P$, then it is also conformally embedded at $P$ in any simply connected domain $D$ satisfying $D_{0} \subset D \subset D_{\infty}$.

Proof. By the very definition, the property of being embedded conformally at a prime end is conformally invariant. Therefore we may assume that $D_{\infty}=\mathbb{D}$. Let $\phi_{0}$ and $\phi$ be any conformal mappings of $\mathbb{D}$ onto $D_{0}$ and $D$, respectively. By the definition of the conformal embedding at a prime end, the point $\sigma_{0} \in \mathbb{T}$ that corresponds to the prime end $P$ under $\phi_{0}$ is a regular contact point of $\phi_{0}$. Denote by $\gamma$ the arc $\phi_{0}\left(\left[0, \sigma_{0}\right)\right)$. Since by hypothesis $D_{0} \subset D \subset \mathbb{D}$, this arc is a slit in $D$. Therefore, by Koebe's Theorem (see, e.g., $\left[22\right.$, Theorem 1, §II.3] or $\left[32\right.$, Lemma 2 on p. 162]) the $\operatorname{arc} C:=\phi^{-1}(\gamma)$ lands on $\mathbb{T}$ at some point $\sigma \in \mathbb{T}$. Consider the function $\varphi:=\phi^{-1} \circ \phi_{0}$. Clearly, $\varphi$ maps $\mathbb{D}$ conformally into itself. Moreover, since by construction $\phi(C)=\gamma$ and $\varphi\left(\left[0, \sigma_{0}\right)\right)=C$ and since the angular derivative $\phi_{0}^{\prime}\left(\sigma_{0}\right)$ exists finitely and does not vanish,

$$
\begin{aligned}
& \lim _{r \rightarrow 1^{-}} \varphi\left(r \sigma_{0}\right)=\sigma, \quad \text { and } \\
& \lim _{r \rightarrow 1^{-}} \frac{\varphi\left(r \sigma_{0}\right)-\sigma}{r \sigma_{0}-\sigma_{0}}=\phi_{0}^{\prime}\left(\sigma_{0}\right)\left(\lim _{C \ni z \rightarrow \sigma} \frac{\phi(z)-\phi_{0}\left(\sigma_{0}\right)}{z-\sigma}\right)^{-1} .
\end{aligned}
$$

By (5.1), $\varphi$ has a contact point at $\sigma_{0}$. It follows, see e.g. [30, Proposition 4.13], that the limit (5.2) exists and belongs to $\overline{\mathbb{C}} \backslash\{0\}$. Hence the limit in the right-hand side of (5.2) also exists and must be finite. By Lemma 4.1 combined with [30, Proposition 4.13], it is, moreover, different from zero. Thus the limit (5.2) is finite, i.e., $\varphi$ has a regular contact point at $\sigma_{0}$. This proves the lemma.

Proof of Theorem 1.2. Clearly, we may assume that $\sigma=1$. Fix any point $w_{0} \in \phi(\mathbb{D})$. By Lemma 5.7 there exists an L-admissible family $\left(D_{t}\right)$ such that $D_{0}=\phi(\mathbb{D}), D_{t_{0}}=\mathbb{D}$, $D_{t} \subset D_{\infty}:=\{z: \operatorname{Re} z<1\}$ for all $t \geq 0$.

Note that, $\left(D_{t}\right)$ is in fact chordally admissible. Indeed, let $P$ be the prime end of $D_{0}$ that corresponds to 1 under the mapping $\phi$. The function $\varphi:=\phi_{\infty}^{-1} \circ \phi$, where $\phi_{\infty}(z):=$ $2 z /(1+z)$ maps conformally $\mathbb{D}$ onto $D_{\infty}$, has a BRFP at 1 , because $\phi$ has a BRFP at 1 by hypothesis and $\phi_{\infty}^{-1}$ has also a fixed point at 1 , being holomorphic there. Then by Lemma $5.9, D_{0}$ is embedded in all $D_{t}$ 's conformally at $P$, which proves our claim.

Hence by Proposition 5.2 and Remark 5.2, there exists a Loewner chain $\left(f_{t}\right)$ such that:

(a) conditions (IIa), (IIb), and (IIc) from Proposition 5.2 are fulfilled;

(b) the point 1 corresponds under the map $f_{0}: \mathbb{D} \rightarrow D_{0}$ to the prime end $P$.

In particular, $f_{t_{0}} \in \operatorname{Möb}(\mathbb{D})$. It follows that, replacing $\left(f_{t}\right)$ if necessary with the Loewner chain $\left(f_{t} \circ f_{t_{0}}^{-1}\right)_{t \geq 0}$, we may assume that:

(c) $f_{t_{0}}=\mathrm{id}_{\mathbb{D}}$ 
(d) $f_{0}(\mathbb{D})=\phi(\mathbb{D}) \subset \mathbb{D}$, and $\varphi_{0, t_{0}}=f_{0}$ has a BRFP at 1 , with $\varphi_{0, t_{0}}^{\prime}(1)=\phi^{\prime}(1)$, where $\left(\varphi_{s, t}\right)$ stands for the evolution family of the Loewner chain $\left(f_{t}\right)$.

Therefore, there exists $\ell \in \operatorname{Möb}(\mathbb{D})$ of the form $\ell=p_{0}^{-1} \circ\left(w \mapsto w+i v_{0}\right) \circ p_{0}$, where $p_{0}(z):=(1+z) /(1-z)$ is the Cayley map of $\mathbb{D}$ onto $\mathbb{H}:=\{w: \operatorname{Re} w>0\}$ sending 1 to $\infty$, such that $\phi=\varphi_{0, t_{0}} \circ \ell$. Define now $\ell_{t}:=p_{0}^{-1} \circ\left(w \mapsto w+i t v_{0} / t_{0}\right) \circ p_{0}$ for all $t \geq 0$. Then, by [14, Lemma 2.8] the family $\left(\psi_{s, t}\right)$ defined by $\psi_{s, t}:=\ell^{-1} \circ \ell_{t} \circ \varphi_{s, t} \circ \ell_{t}^{-1} \circ \ell$ for all $t \geq s \geq 0$, is an evolution family in $\mathbb{D}$. Note that $\ell_{0}=\mathrm{id} \mathbb{D}$ and $\ell_{t_{0}}=\ell$ by construction. Thus $\psi_{0, t_{0}}=\varphi_{0, t_{0}} \circ \ell=\phi$. The proof is now complete.

\section{Examples And Remarks on Theorem 1.1}

Theorem 1.1 contains a characterization of boundary regular fixed points of an evolution family in terms of the associated Loewner chain assuming that one of the functions of the Loewner chain is conformal at a certain boundary point. A similar result can be obtained when the function has a simple pole. Let us recall that a function $f: \mathbb{D} \rightarrow \mathbb{C}$ has a simple pole (in the angular sense) at a boundary point $\sigma \in \mathbb{T}$ if $\angle \lim _{z \rightarrow \sigma}(z-\sigma) f(z) \in \mathbb{C}^{*}$.

Following the notation in the classical case, we call this limit the residue of $f$ at $\sigma$ and denote it by $\operatorname{Res}(f ; \sigma)$.

Corollary 6.1. Let $\left(f_{t}\right)$ be a Loewner chain and $\left(\varphi_{s, t}\right)$ its evolution family. Suppose there exists $t_{0} \geq 0$ such that the map $f_{t_{0}}$ has a simple pole at a point $\sigma \in \mathbb{T}$. Then the following two conditions are equivalent:

(i) for every $s \geq 0$ the following assertions hold:

(i.1) the map $f_{s}$ has a simple pole at the point $\sigma$;

(i.2) $\limsup _{t \rightarrow s^{+}}\left|\arg \left(\operatorname{Res}\left(f_{s} ; \sigma\right) / \operatorname{Res}\left(f_{t} ; \sigma\right)\right)\right|<\pi$.

(ii) the evolution family $\left(\varphi_{s, t}\right)$ has a boundary regular fixed point at $\sigma$.

Moreover, if conditions (i) and (ii) above hold, then

(iii) the function $t \mapsto \operatorname{Res}\left(f_{t} ; \sigma\right)$ is locally absolutely continuous on $[0,+\infty)$, with $\arg \left(\operatorname{Res}\left(f_{t} ; \sigma\right)\right)$ being constant.

Proof. Let us fix $T>t_{0}$ and a point $w_{0} \notin f_{T}(\mathbb{D})$. Write $l(t):=T t /(1+t)$ and consider the family of univalent functions $g_{t}: \mathbb{D} \rightarrow \mathbb{C}$ given by $z \mapsto g_{t}(z):=1 /\left(f_{l(t)}(z)-w_{0}\right)$, for all $t \geq 0$ and $z \in \mathbb{D}$. It is clear that $f_{l(t)}$ has a simple pole at $\sigma$ if and only if $g_{t}(\sigma)=0$ and $g_{t}$ is conformal at $\sigma$. In such a case, $g_{t}^{\prime}(\sigma)=1 / \operatorname{Res}\left(f_{t} ; \sigma\right)$. On the other hand, the evolution family associated with the Loewner chain $\left(g_{t}\right)$ is nothing but $\left(\varphi_{l(s), l(t)}\right)$. Bearing in mind these remarks and applying Theorem 1.1 to the Loewner chain $\left(g_{t}\right)$ one can easily complete the proof.

Remark 6.2. Thanks to the chain rule for angular derivatives, see, e.g., [19, Lemma 2], and condition EF2 in the definition of evolution families, hypothesis (A) in Theorem 1.1 is equivalent to 
$\left(\mathrm{A}^{\prime}\right)$ for each $t \geq 0$ the point $\sigma$ is a BRFP of $\left(\varphi_{0, t}\right)$.

Now we consider a family of examples demonstrating that conditions and conclusions in Theorem 1.1 are the best possible in some sense.

It easily follows from the definition that if $G$ is a Herglotz vector field, then $t \mapsto G^{\prime}(z, t)$ is locally integrable on $[0,+\infty)$ for any $z \in \mathbb{D}$. This property is not inherited when passing to the boundary:

Example 6.3. We construct an example of a Herglotz vector field having a BRNP at 1 for a.e. $t \geq 0$ such that the dilation $\lambda(t):=G^{\prime}(1, t)$ is not locally integrable. We actually show that any non-negative measurable function $\lambda$ defined a.e. on $[0,+\infty)$ can arise in this way.

Let $r$ and $\lambda$ be measurable non-negative functions defined a.e. on $[0,+\infty)$. Assume that $r(t)<1$ for a.e. $t \geq 0$ and that $M(t):=\lambda(t)(1-r(t))$ is locally integrable on $[0,+\infty)$. Clearly, given any non-negative measurable function $\lambda$ one can find a function $r$ such that these requirements are met.

Denote by $p_{0}(z):=(1+z) /(1-z)$ and consider the function

$$
G_{\lambda, r}(z, t):=(1-z)^{2} \frac{\lambda(t)}{2}\left(p_{0}(r(t) z)-p_{0}(z)\right)
$$

for a.e. $t \geq 0$ and all $z \in \mathbb{D}$. By Theorem 2.8, for a.e. $t \geq 0, G_{\lambda, r}(\cdot, t)$ is an infinitesimal generator with a BRNP of dilation $\lambda(t)$ at 1 . Moreover, a simple calculation shows that

$$
p_{0}(r z)-p_{0}(z)=-\frac{2(1-r) z}{(1-z)(1-r z)} .
$$

Therefore, $\left|G_{\lambda, r}(z, t)\right| \leq 2 M(t)$ for a.e. $t \geq 0$.

Thus, $G_{\lambda, r}$ is a Herglotz vector field having, for a.e. $t \geq 0$, a BRNP of dilation $\lambda(t)$ at 1 . Since we can choose any measurable non-negative function for $\lambda$, this example shows that condition (B.2) in Theorem 1.1 does not follow from condition (B.1).

Remark 6.4. Note that if an evolution family $\left(\varphi_{s, t}\right)$ has a BRFP at $\sigma \in \partial \mathbb{D}$ with nondecreasing spectral function $\Lambda$, which means that $\sigma$ is the DW-point of all $\varphi_{s, t}$ 's different from the identity mapping, and if the evolution family $\left(\varphi_{s, t}\right)$ is of some order $d \in[1,+\infty]$, then $\Lambda^{\prime}$ must be of class $L_{\text {loc }}^{d}$, see [8, Theorem 7.3].

The construction in Example 6.3 can be also used to see that in contrast to the case of boundary DW-point (see Remark 6.4), any non-positive locally integrable function can arise as the derivative of the spectral function of an evolution family $\left(\varphi_{s, t}\right)$ at a BRFP which is not the DW-point even if we require that the evolution family $\left(\varphi_{s, t}\right)$ is of the best possible order $d=+\infty$.

Example 6.5. In the construction of Example 6.3, assume additionally that $\lambda$ is of class $L_{\text {loc }}^{1}$. Set

$$
r(t):= \begin{cases}0, & \text { if } \lambda(t) \leq 1 \\ 1-1 / \lambda(t), & \text { if } \lambda(t) \geq 1\end{cases}
$$


Then $0 \leq M(t) \leq 1$ for a.e. $t \geq 0$. Hence $G_{\lambda, r}$ is an $L^{\infty}$-Herglotz vector field. By Theorem 1.1, the $L^{\infty}$-evolution family $\left(\varphi_{s, t}\right)$ generated by $G_{\lambda, r}$ has a BRFP point at 1 and its spectral function $\Lambda$ satisfies $\Lambda^{\prime}=-\lambda$ a.e. on $[0,+\infty)$. Therefore, the regularity of the spectral function in Theorem 1.1 does not depend on the order of the evolution family.

Nevertheless, as the following proposition shows, the order of $\left(\varphi_{s, t}\right)$ still imposes certain restrictions on the behaviour of $\Lambda$.

Proposition 6.6. Let $\left(\varphi_{s, t}\right)$ be an evolution family of order $d \in[1,+\infty]$ and $G$ its Herglotz field. Suppose that $\sigma \in \mathbb{T}$ is a BRFP of $\left(\varphi_{s, t}\right)$ and let $\lambda(t):=G^{\prime}(\sigma, t)$. Then $\lambda^{-}:=$ $\frac{1}{2}(|\lambda|-\lambda) \in L_{\text {loc }}^{d}([0,+\infty), \mathbb{R})$.

Proof. Let $A:=\{t \geq 0: \lambda(t) \leq 0\}$. Since $\lambda$ is measurable, it is easy to check that $\widehat{G}(z, t):=G(z, t) \chi_{A}(t)$ is a Herglotz vector field of order $d$. Moreover, for a.e. $t \geq 0, \widehat{G}$ has a BRNP at $\sigma$ with the dilation $-\lambda^{-}(t)$. By Theorem 1.1 the evolution family $\left(\widehat{\varphi}_{s, t}\right)$ of order $d$ generated by $\widehat{G}$ has a RBFP at $\sigma$ with the spectral function $\widehat{\Lambda}(t):=\int_{0}^{t} \lambda^{-}(s) d s$, which is non-decreasing on $[0,+\infty)$. Therefore, $\lambda^{-} \in L_{\text {loc }}^{d}([0,+\infty), \mathbb{R})$ by Remark 6.4 .

The previous examples rise naturally the question about the behavior of the evolution family at the BRNP of its Herglotz vector field if the dilation $\lambda$ is not locally integrable. Our next two examples reveal two different types of behavior: in the first case this point is not a fixed point and is mapped into $\mathbb{D}$ by $\varphi_{0, t}$ whenever $t>0$, while in the second case this point is still a boundary fixed point of all $\varphi_{s, t}$ 's, although it is not regular if $s=0$.

Example 6.7. Let us consider again the vector field $G_{\lambda, r}$ from Example 6.3 with a particular choice of the function $\lambda$. Namely, we fix some $T>0$ and assume that $r:(0, T) \rightarrow(0,1)$ is a smooth function that tends to 1 as $t \rightarrow 0^{+}$. Set $\lambda(t):=2 /(1-r(t))$ for all $t \in(0, T)$. (For $t \geq T$ we can extend the functions $r$ and $\lambda$ assuming them to be constants.)

Then again $G_{\lambda, r}$ is an $L^{\infty}$-Herglotz vector field. Let us consider the generalized Loewner-Kufarev equation with $G=G_{\lambda, r}$ restricted to $(0,1)$. The solution $\xi_{x}$ to

$$
\frac{d \xi_{x}(t)}{d t}=G_{\lambda, r}\left(\xi_{x}(t), t\right)=-\left.\frac{2 z(1-z)}{1-r(t) z}\right|_{z:=\xi_{x}(t)}, t \geq 0, \quad \xi_{x}(0)=x \in(0,1),
$$

stays in $(0,1)$ for all $t \geq 0$, because $G_{\lambda, r}(\xi)$ is real for $\xi \in(0,1)$ and $G_{\lambda, r}(0)=0$.

If we let

$$
r(t):=1-\left(\frac{2}{\alpha}-1\right)\left(e^{\alpha t}-1\right)
$$

for fixed $\alpha \in(0,2)$, the function $\xi_{*}(t):=e^{-\alpha t}$, which tends to $1^{-}$as $t \rightarrow 0^{+}$, is a solution to $(6.2)$ on $(0, T)$ for $T>0$ small enough. Thus, we have $\xi_{x}(t)<\xi_{*}(t)$ for all $t \in(0, T)$ and all $x \in(0,1)$. Indeed, otherwise there would exist $t^{*} \in(0, T)$ and $x^{*} \in(0,1)$ such that $\xi_{x^{*}}\left(t^{*}\right)=\xi_{*}\left(t^{*}\right)$, which contradicts the uniqueness property for the solutions to the 
Cauchy problem (6.2). Thus the point 1 is not a boundary fixed point of $\varphi_{0, t}$ for any $t>0$, although $\varphi_{0, t}(1)$ exists in the angular sense and lies in $(0,1)$.

Example 6.8. In the previous example, for small $t>0$ the function $r(t)$ behaved asymptotically as $1-(2-\alpha) t$. Let us now consider the same choice of $\lambda$ but for the case when $r(t)=1-\beta t$ for all $t \in(0, T)$, where $\beta>2$ is fixed. We are going to prove that in this case $\varphi_{s, t}$ has a boundary fixed point at 1 . Note that in view of Theorem 1.1, this point cannot be a BRFP whenever $s=0$, because $\lambda(t)=1 /(\beta t)$ for all $t \in(0, T)$.

First we show that there are no non-trivial singular solutions, i.e., the problem

$$
\frac{d \xi_{*}(t)}{d t}=G_{\lambda, r}\left(\xi_{*}(t), t\right), t \in(0, T), \quad \lim _{t \rightarrow 0^{+}} \xi_{*}(t)=1,
$$

has no solutions $\xi_{*}:(0, T) \rightarrow(0,1)$. Suppose on the contrary that such a solution exists. Denote $u(t):=\left(1-\xi_{*}(t)\right) / t$. Then according to Lagrange's Mean Value Theorem applied to the function $\xi_{*}$ on the interval $(0, t),(6.3)$ implies that for all $t \in(0, T)$,

$$
0 \leq u(t)=-G_{\lambda, r}\left(\xi_{*}\left(t^{*}\right), t^{*}\right) \leq \frac{2 u\left(t^{*}\right)}{\beta+r\left(t^{*}\right) u\left(t^{*}\right)}
$$

where $t^{*} \in(0, t)$ depends on $t$. Assuming for convenience that $T>0$ is small enough so that $r(t)>1 / 2$ for all $t \in(0, T)$, we now conclude that $0 \leq \alpha:=\sup _{t \in(0, T)} u(t) \leq 4$. Recalling that $\beta>2$ and using the above inequality again, we see that actually $\alpha=0$ and hence $\xi_{*} \equiv 1$.

Note that $\varphi_{s, t}$ extends holomorphically to $\mathbb{T}$ for any $s>0$ and $t \geq s$. Hence to show that 1 is a boundary fixed point of $\varphi_{s, t}$ for all $t \geq s \geq 0$, it is clearly sufficient to prove this statement for $s=0$ and $t \in(0, T)$. To this end, in turn, it is sufficient to show that $\sup _{x \in(0,1)} \varphi_{0, t_{0}}(x)=1$ for at least one $t_{0} \in(0, T)$.

Suppose on the contrary that $m(t):=\sup _{x \in(0,1)} \varphi_{0, t}(x)<1$ for all $t \in(0, T)$. Note that the function $m$ does not increase on $(0, T)$. Then using Lebesgue's Dominated Convergence Theorem we can pass to the limit as $x \rightarrow 1^{-}$under the integral sign in the equality $\varphi_{0, t}(x)=\varphi_{0, t_{0}}(x)+\int_{t_{0}}^{t} G_{\lambda, r}\left(\varphi_{0, s}(x), s\right) d s$, which holds for any $t, t_{0} \in(0, T)$ and all $x \in(0,1)$, to conclude that $\xi_{*}(t):=\lim _{x \rightarrow 1^{-}} \varphi_{0, t}(x)$ is a solution to problem (6.3). (The limit exists because $\varphi_{0, t}$ is increasing and bounded on $(0,1)$.) However, as we proved above there exist no non-trivial solutions to (6.3).

Thus in this example the point 1 is a boundary (non-regular) fixed point of the evolution family $\left(\varphi_{s, t}\right)$ and a BRNP of the Herglotz vector field $G(\cdot, t)=G_{\lambda, r}(\cdot, t)$ of $\left(\varphi_{s, t}\right)$ for a.e. $t \geq 0$, although the dilation $\lambda(t)=G^{\prime}(t, 1)$ is not locally integrable.

Remark 6.9. In $[15, \S 7]$ it was constructed an example of an evolution family $\left(\varphi_{s, t}\right)$ with the DW-point at 1 such that all elements of every Loewner chain $\left(f_{t}\right)$ associated with $\left(\varphi_{s, t}\right)$ have no angular limits at 1 . Hence the conformality of $f_{t_{0}}$ at $\sigma$ is an essential condition for assertion (C) to be included in Theorem 1.1. 


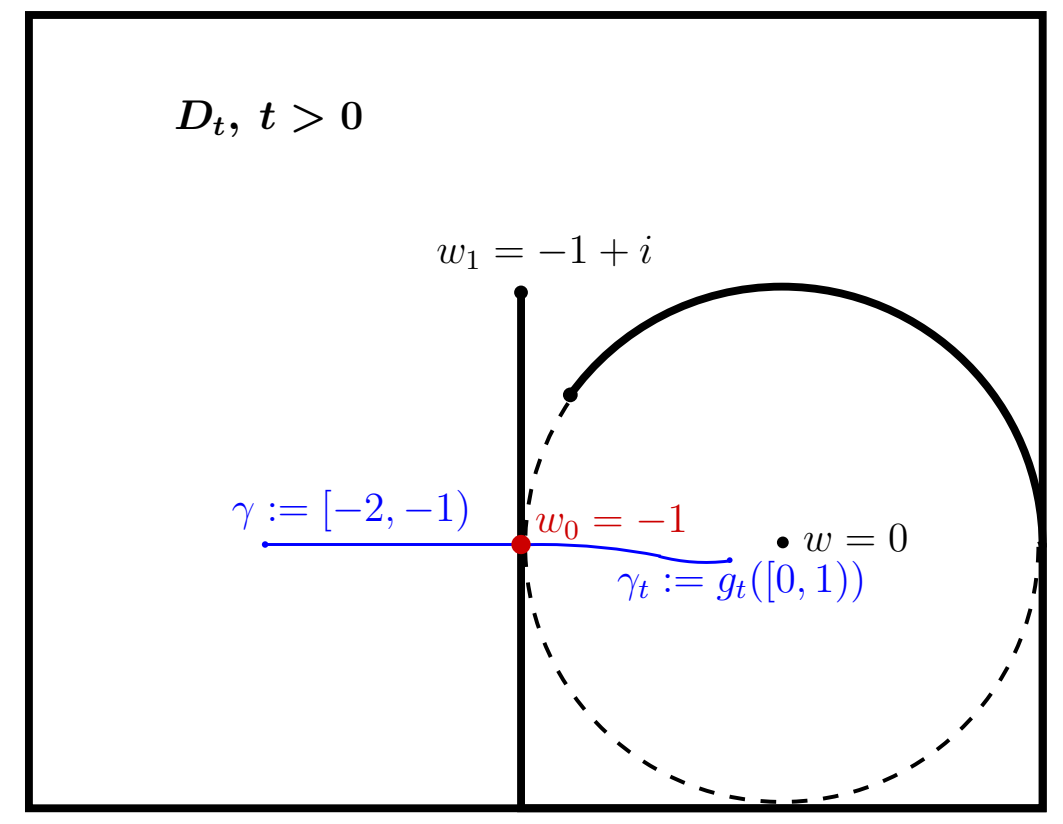

Figure 1. The inclusion chain $\left(D_{t}\right)$.

Now we present an example showing that condition (C.3) in Theorem 1.1 cannot be omitted.

Example 6.10. We are going to construct a Loewner chain satisfying conditions (C.1) and (C.2) from Theorem 1.1 but not (C.3), for which (A) does not hold, i.e. a Loewner chain $\left(f_{t}\right)$ such that all $f_{t}$ 's are conformal at the point 1 , share the same value at this point (in our construction $f_{t}$ 's have continuous extension to $\left.\overline{\mathbb{D}}\right)$, but the evolution family $\left(\varphi_{s, t}\right)$ of $\left(f_{t}\right)$ fails to have a BRFP at 1 . The construction is divided into several steps.

Step 1. First we construct an inclusion chain $\left(D_{t}\right)$ composed of domains $D_{t}$ with locally connected boundaries such that $D_{0}$ is embedded in each of $D_{t}$ 's conformally at some prime end $P$, whose impression $\left\{w_{0}=-1\right\}$ corresponds to only one boundary accessible point of $D_{0}$ and to exactly two boundary accessible points of $D_{t}$ for all $t>0$.

$$
\text { Let }
$$

$$
\begin{aligned}
D_{0}:= & \{x+i y:-1<x<1,-1<y<0\} \cup \mathbb{D}, \\
D_{t}:= & \{x+i y:-3<x<1,-1<y<2\} \backslash([-1-i,-1+i] \\
& \left.\cup\left\{z:|z|=1,0 \leq \arg z \leq \pi\left(1-e^{-t}\right)\right\}\right) \quad \text { for all } t>0,
\end{aligned}
$$

see Figure 1. Clearly, $\left(D_{t}\right)_{t \geq 0}$ is an inclusion chain. Since $D_{0}$ is a Jordan domain, there is a one-to-one correspondence between $\partial D_{0}$ and the set of prime ends of $D_{0}$. Denote by $P$ the 
prime end corresponding to the boundary point $w_{0}=-1$. Using [30, Theorem 3.9, p. 52] with $\alpha=1$ and the Schwarz Reflection Principle, it is easy to see that $D_{0}$ is embedded conformally in $D_{t}$ at $P$ for any $t \geq 0$.

Step 2. Now we construct a Loewner chain $\left(\tilde{g}_{t}\right)$ such that $\tilde{g}_{t}(0)=0$ and $\tilde{g}_{t}(\mathbb{D})=D_{t}$ for all $t \geq 0$, the evolution family $\left(\tilde{\psi}_{s, t}\right)$ of $\left(\tilde{g}_{t}\right)$ has a BRFP at 1 , and for every $t>0$ there exists $\sigma(t) \in \mathbb{T} \backslash\{1\}$ such that $\tilde{g}_{t}(\sigma(t))=\tilde{g}_{t}(1)=-1$.

By [15, Theorem 1.10], there exists a Loewner chain $g_{t}$ of chordal type such that up to the change of the parametrization of the family $\left(D_{t}\right)$, we have $g_{t}(\mathbb{D})=D_{t}$. In what follows we assume that $\left(D_{t}\right)$ is reparameterized in such a way. By the very definition of the Loewner chain of chordal type, all elements of the evolution family $\left(\psi_{s, t}\right)$ associated with $\left(g_{t}\right)$, different from $\mathrm{id}_{\mathbb{D}}$, share the same DW-point $\tau=1 \in \mathbb{T}$. Moreover, by the construction used in the proof of [15, Theorem 1.10], $g_{t}(1)=w_{0}=-1$ for all $t \geq 0$. Here we have taken into account the fact that $\partial D_{t}$ is locally connected, so by Carathéodory's Continuous Extension Theorem, see e.g. [30, Theorem 2.1 on p. 20], the function $g_{t}$ has a continuous extension to $\overline{\mathbb{D}}$, which we will denote again by $g_{t}$.

Since $\left(\psi_{s, t}\right)$ has a BRFP at $\tau=1$, by Theorem 1.1, $\arg g_{t}(1)$ does not depend on $t$. Therefore, for each $t>0$, the slits $\gamma_{t}:=g_{t}([0,1))$ and $\gamma:=[-2,-1)$ in $D_{t}$, which land both at $w_{0}=-1$, are not equivalent. This means (see e.g. [22, Theorem 1, §II.3]) that there is a point $\sigma(t) \in \mathbb{T} \backslash\{\tau=1\}$ such that $g_{t}(\sigma(t))=w_{0}=-1$. The same holds for the Loewner chain $\left(\tilde{g}_{t}\right)$ defined by $\tilde{g}_{t}:=g_{t} \circ \ell_{t}$ for all $t \geq 0$, where $\left(\ell_{t}\right) \subset \operatorname{Möb}(\mathbb{D})$ is given by

$$
\ell_{t}(z):=\frac{1+\overline{z_{0}(t)}}{1+z_{0}(t)} \frac{z+z_{0}(t)}{1+z \overline{z_{0}(t)}}, \quad z_{0}(t):=g_{t}^{-1}(0), t \geq 0 .
$$

To see that $\left(\tilde{g}_{t}\right)$ is really a Loewner chain and, correspondingly, $\left(\tilde{\psi}_{s, t}\right)=\left(\tilde{g}_{t}^{-1} \circ \tilde{g}_{s}\right)=$ $\left(\ell_{t}^{-1} \circ \psi_{s, t} \circ \ell_{s}\right)$ is its evolution family it is sufficient to apply [14, Lemmas 2.8 and 3.2] bearing in mind that, by [8, Proposition 3.7], the function $t \mapsto z_{0}(t)=\psi_{0, t}\left(z_{0}(0)\right)$ is locally absolutely continuous.

Step 3. Now we show that $0<t \mapsto \sigma(t)$ has a locally absolutely continuous extension to $[0,+\infty)$.

Fix $s_{0}>0$ and consider the evolution family formed by the function $\hat{\psi}_{s, t}:=\tilde{\psi}_{s_{0}+s, s_{0}+t}$, $0 \leq s \leq t$. The functions $\hat{g}_{t}:=\tilde{g}_{s_{0}+t}, t \geq 0$, form a Loewner chain associated with $\left(\hat{\psi}_{s, t}\right)$. Using the Schwarz Reflection Principle it then easy to see that the evolution family $\left(\hat{\psi}_{s, t}\right)$ has a regular contact point at $\sigma\left(s_{0}\right)$. Since by construction $\sigma\left(s_{0}+t\right)=\hat{\psi}_{0, t}\left(\sigma\left(s_{0}\right)\right)$ for all $t>0$ and since we may choose any $s_{0}>0$, Theorem 3.5 implies that $(0,+\infty) \ni t \mapsto \sigma(t)$ is locally absolutely continuous. To prove that this function can be extended absolutely continuous to $[0,+\infty)$ it is sufficient to show that $\arg \sigma(t)$ is monotonic.

By a similar argument one can prove that the unique preimage $\sigma_{1}(t)$ of the point $w_{1}:=-1+i$ w.r.t. $\tilde{g}_{t}, t>0$, depends on $t$ (locally absolutely) continuous. Denote $L_{t}:=$ $g_{t}^{-1}\left(\left[w_{0}, w_{1}\right]\right), t>0$. Then, see e.g. [30, Proposition 2.5 on p. 23], $L_{t}$ is the arc of the unit 
circle with end-points $\tau=1$ and $\sigma(t)$ containing $\sigma_{1}(t)$ as an interior point. Note that [15, Theorem 8.1], the functions $\tilde{\psi}_{s, t}$ have continuous extension to $\overline{\mathbb{D}}$. Then by construction $\tilde{\psi}_{s, t}\left(L_{s}\right)=L_{t}$. Note also that $\tilde{\psi}_{s, t}(0)=0$. Therefore, by Loewner's Lemma (see e.g. [30, Proposition 4.15 on p. 85]) the length of $L_{s}$ does not exceed the length of $L_{t}$ whenever $0<s \leq t$. Bearing in ming that $(0,+\infty) \mapsto \sigma_{1}(t) \in L_{t} \backslash\{\sigma(t), \tau=1\}$ is continuous, we easily conclude that $t \mapsto \arg \sigma(t)$ is a monotonic function.

Step 4. We prove that $\sigma(t) \rightarrow 1$ as $t \rightarrow 0^{+}$.

Recall that $\tilde{g}_{t}$ is univalent in $\mathbb{D}$ for any $t \geq 0$. Note also that by construction the Euclidean

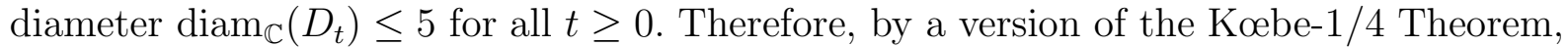
see e.g. [29, Corollary 1.4 on p. 22] for any $z \in \mathbb{D}$ and any $t \geq 0$,

$$
\frac{\left|\tilde{g}_{t}^{\prime}(z)\right|}{1+\left|\tilde{g}_{t}(z)\right|^{2}}\left(1-|z|^{2}\right) \leq\left|\tilde{g}_{t}^{\prime}(z)\right|\left(1-|z|^{2}\right) \leq 4 \operatorname{dist}\left(\tilde{g}_{t}(z), \partial D_{t}\right) \leq 20 .
$$

Again by construction, for each $t>0$ there exists an $\operatorname{arc} C_{t}$ of the unit circle with the following properties:

(a) for each $t>0, C_{t}$ is a cross-cut in $D_{t}$ and one of the landing points of $C_{t}$ coincides with $w_{0}=-1$;

(b) for each $t>0, C_{t}$ separates $\left(w_{0}, w_{1}\right]$ from $\tilde{g}_{t}(0)=0$, i.e. the closure of the connected component of $D_{t} \backslash C_{t}$ that contains $\tilde{g}_{t}(0)$ does not intersect $\left(w_{0}, w_{1}\right]$;

(c) $\operatorname{diam}_{\mathbb{C}}\left(C_{t}\right) \rightarrow 0$ as $t \rightarrow 0^{+}$.

By the Lehto - Virtanen version of the No-Kœbe-Arcs Theorem, see, e.g., [29, Theorem 9.2 and Remark on p.265], from (6.4) and (c) it follows that we have:

(d) $\operatorname{diam}_{\mathbb{C}}\left(\tilde{g}_{t}^{-1}\left(C_{t}\right)\right) \rightarrow 0$ as $t \rightarrow 0^{+}$.

Now from (b) and (d) it follows that $\operatorname{diam}_{\mathbb{C}}\left(L_{t}\right) \rightarrow 0$ as $t \rightarrow 0^{+}$and thus our claim is proved.

Step 5. Finally, we construct the desired Loewner chain $\left(f_{t}\right)$.

By Steps 3 and 4 the function $t \mapsto \sigma(t)$ extended to $t=0$ by $\sigma(0):=1$ is locally absolutely continuous on $[0,+\infty)$. Therefore, the formula $f_{t}(z):=\tilde{g}_{t}(\sigma(t) z)$ for all $z \in \mathbb{D}$ and all $t \geq 0$ defines a Loewner chain $\left(f_{t}\right)_{t \geq 0}$. Moreover, by construction for all $t \geq 0$ the function $f_{t}$ is conformal at 1 and $f_{t}(1)=w_{0}=-1$, i.e. the Loewner chain $\left(f_{t}\right)$ fulfills conditions (C.1) and (C.2) in Theorem 1.1, but the evolution family $\left(\varphi_{s, t}\right)$ of $\left(f_{t}\right)$ does not fulfill condition (A) in Theorem 1.1, since $\varphi_{0, t}(1)=\sigma(t) \neq 1$ for all $t>0$. The reason is that condition (C.3) does not hold: $\arg f_{t}^{\prime}(1)=0$ for all $t>0$, while $\arg f_{0}^{\prime}(1)=\pi$.

\section{REFERENCES}

[1] M. Abate, Iteration theory of holomorphic maps on taut manifolds, Mediterranean Press, Rende, 1989.

[2] M. Abate, F. Bracci, M. D. Contreras, S. Díaz-Madrigal, The evolution of Loewner's differential equations Newsletter European Math. Soc. 78, December 2010, 31-38. 
[3] L.V. Ahlfors, Conformal invariants, reprint of the 1973 original, AMS Chelsea Publishing, Providence, RI, 2010.

[4] I.A. Aleksandrov, Parametric continuations in the theory of univalent functions (in Russian), Izdat. "Nauka", Moscow, 1976.

[5] L. Arosio, F. Bracci, H. Hamada, G. Kohr, An abstract approach to Loewner's chains. J. Anal. Math., to appear.

[6] E. Berkson, H. Porta, Semigroups of holomorphic functions and composition operators, Michigan Math. J. 25 (1978), 101-115.

[7] F. Bracci, Holomorphic evolution: metamorphosis of the Loewner equation. Boll. Unione Mat. Ital. Sez. B Artic. Ric. Mat., to appear.

[8] F. Bracci, M. Contreras, S. Díaz-Madrigal, Evolution Families and the Loewner Equation I: the unit disc. J. Reine Angew. Math. (Crelle's Journal), 672 (2012), 1-37.

[9] F. Bracci, M. D. Contreras, S. Díaz-Madrigal, Evolution Families and the Loewner Equation II: complex hyperbolic manifolds. Math. Ann. 344 (2009), No.4, 947-962.

[10] F. Bracci, M. D. Contreras, S. Díaz-Madrigal, Pluripotential theory, semigroups and boundary behavior of infinitesimal generators in strongly convex domains. J. Eur. Math. Soc. 12 (2010), 23-53.

[11] F. Bracci, M. Contreras, S. Díaz-Madrigal, Aleksandrov-Clark measures and semigroups of analytic functions in the unit disc. Ann. Acad. Sci. Fenn. 33 (2008), 231-240.

[12] E.F. Collingwood, A.J. Lohwater, The theory of cluster sets, Cambridge Tracts in Mathematics and Mathematical Physics, No. 56 Cambridge Univ. Press, Cambridge, 1966. MR0231999 (38 \#325)

[13] M. D. Contreras, S. Díaz-Madrigal, Analytic flows on the unit disk: angular derivatives and boundary fixed points. Pacific J. Math. 222 (2005), No. 2, 253-286.

[14] M. D. Contreras, S. Díaz-Madrigal, P. Gumenyuk, Loewner chains in the unit disk. Rev. Mat. Iberoam. 26 (2010), 975-1012.

[15] M. D. Contreras, S. Díaz-Madrigal, P. Gumenyuk, Geometry behind chordal Loewner chains, Complex Anal. Oper. Theory 4 (2010), No. 3, 541-587. MR2719792 (2011h:30037)

[16] M. D. Contreras, S. Díaz-Madrigal, P. Gumenyuk, Loewner Theory in annulus I: evolution families and differential equations, Trans. Amer. Math. Soc. 365 (2013), No. 5, 2505-2543.

[17] M. D. Contreras, S. Díaz-Madrigal, P. Gumenyuk, Local duality in Loewner equations. Preprint, 2012, 33pp. Institut Mittag-Leffler ISRN IML-R- -16-11/12- -SE+fall; arXiv:1202.2334 [math.CV].

[18] M. D. Contreras, S. Díaz-Madrigal, Ch. Pommerenke, Fixed points and boundary behaviour of the Koenings function. Ann. Acad. Sci. Fenn. Math. 29 (2004), 471-488.

[19] M. D. Contreras, S. Díaz-Madrigal, Ch. Pommerenke, On boundary critical points for semigroups of analytic functions. Math. Scand. 98 (2006), No. 1, 125-142.

[20] C.C. Cowen, Ch. Pommerenke, Inequalities for the angular derivative of an analytic function in the unit disk. J. London Math. Soc. (2), 26 (1982), 271-289.

[21] M. Elin, D. Shoikhet, Semigroups of holomorphic mappings with boundary fixed points and spirallike mappings. Geometric function theory in several complex variables, 82-117, World Sci. Publ., River Edge, NJ, 2004.

[22] G.M. Goluzin, Geometric theory of functions of a complex variable, $2^{\text {nd }}$ ed., "Nauka", Moscow, 1966 (in Russian); English transl.: Amer. Math. Soc., 1969.

[23] V.V. Goryainov, O.S. Kudryavtseva, One-parameter semigroups of analytic functions, fixed points and the Koenigs function, Mat. Sb. 202 (2011), No. 7, 43-74 (Russian); translation in Sbornik: Mathematics, 202 (2011), No. 7-8, 971-1000.

[24] I. Graham, G. Kohr, Geometric function theory in one and higher dimensions, Monographs and Textbooks in Pure and Applied Mathematics, 255, Dekker, New York, 2003. 
[25] A.N. Kolmogorov, S.V. Fomin, Introductory real analysis, translated from the second Russian edition and edited by Richard A. Silverman, Dover, New York, 1975. MR0377445 (51 \#13617)

[26] P.P. Kufarev, On one-parameter families of analytic functions, (in Russian) Mat. Sb. 13 (1943), $87-118$.

[27] K. Löwner, Untersuchungen über schlichte konforme Abbildungen des Einheitskreises, Math. Ann. 89 (1923), 103-121.

[28] Ch. Pommerenke, Über dis subordination analytischer funktionen, J. Reine Angew Math. 218 (1965), 159-173.

[29] Ch. Pommerenke, Univalent Functions, Vandenhoeck \& Ruprecht, Göttingen, 1975.

[30] Ch. Pommerenke, Boundary behaviour of conformal mappings, Springer-Verlag, 1992.

[31] S. Reich, D. Shoikhet, Metric domains, holomorphic mappings and nonlinear semigroups, Abstr. Appl. Anal. 3 (1998), No. 1-2, 203-228. MR1700285 (2000f:47096)

[32] J.H. Shapiro, Composition Operators and Classical Function Theory, Springer-Verlag, New York, 1993.

[33] D. Shoikhet, Semigroups in geometrical function theory. Kluwer Academic Publishers, Dordrecht, 2001.

[34] D. Shoikhet, Representations of holomorphic generators and distortion theorems for spirallike functions with respect to a boundary point. Int. J. Pure Appl. Math. 5 (2003), 335-361.

[35] A. G. Siskakis, Semigroups of Composition Operators and the Cesàro Operator on $H^{p}(D), \mathrm{Ph}$. D. Thesis, University of Illinois, 1985.

F. Bracci, P. Gumenyuk: Dipartimento di Matematica, Università di Roma "Tor VerGata", Via Della Ricerca Scientifica 1, 00133, Roma, Italia.

E-mail address: fbracci@mat.uniroma2.it

E-mail address: gumenyuk@mat. uniroma2.it

M. D. Contreras, S. Díaz-Madrigal: Camino de los Descubrimientos, s/n, Departamento de Matemática Aplicada II and IMUS, Universidad de Sevilla, Sevilla, 41092, Spain.

E-mail address: contreras@us.es

E-mail address: madrigal@us.es 